\title{
TWO NEW HAGER-ZHANG ITERATIVE SCHEMES WITH IMPROVED PARAMETER CHOICES FOR MONOTONE NONLINEAR SYSTEMS AND THEIR APPLICATIONS IN COMPRESSED SENSING
}

\author{
Mohammed Yusuf Waziri $I^{1,4} \odot$, Kabiru Ahmed ${ }^{1,4}$, \\ Abubakar SANi Halilu ${ }^{2,4, *} \odot$ AND JAMILU SABI' ${ }^{3,4}$
}

\begin{abstract}
Notwithstanding its efficiency and nice attributes, most research on the Hager-Zhang (HZ) iterative scheme are focused on unconstrained minimization problems. Inspired by this and recent extensions of the one-parameter HZ scheme to system of nonlinear monotone equations, two new HZtype iterative methods are developed in this paper for solving system of monotone equations with convex constraint. This is achieved by developing two HZ-type search directions with new parameter choices combined with the popular projection method. The first parameter choice is obtained by minimizing the condition number of a modified HZ direction matrix, while the second choice is realized using singular value analysis and minimizing the spectral condition number of a nonsingular HZ search direction matrix. Interesting properties of the schemes include solving non-smooth problems and satisfying the inequality that is vital for global convergence. Using standard assumptions, global convergence of the schemes are proven and numerical experiments with recent methods in the literature, indicate that the methods proposed are promising. The effectiveness of the schemes are further demonstrated by their applications to sparse signal and image reconstruction problems, where they outperform some recent schemes in the literature.
\end{abstract}

Mathematics Subject Classification. 90C30, 65K05, 90C53, 49M37, 15A18.

Received February 27, 2021. Accepted December 23, 2021.

\section{INTRODUCTION}

The fields of sciences, engineering, industry and other important areas of human endeavor employ vital applications with models in the form of system of nonlinear equations. Various examples have been considered in this areas in recent years.

Generally, the system of monotone nonlinear equations is formulated by:

$$
g(x)=0, \quad x \in \mathbf{R}^{n},
$$

Keywords. Nonlinear monotone systems, line search, inexact line search, projection operator.

1 Department of Mathematical Sciences, Bayero University, Kano, Nigeria.

2 Department of Mathematics, Sule Lamido University, Kafin Hausa, Nigeria.

3 Department of Mathematics, Northwest University, Kano, Nigeria.

4 Numerical Optimization Research Group, Bayero University, Kano, Nigeria.

*Corresponding author: ashalilu.mth@gmail.com 
where $g: \mathbf{R}^{n} \rightarrow \mathbf{R}^{n}$ represents a continuous nonlinear mapping, which satisfies the monotonicity condition

$$
(g(x)-g(y))^{T}(x-y) \geq 0, \quad \text { for all vectors } x, y \in \mathbf{R}^{n} .
$$

This paper focuses on the constrained version of (1.1), where the vector $x$ lies in a nonempty closed convex set, say $\Omega \subset \mathbf{R}^{n}$. A number of applications involve the monotone equations in (1.1) and its constrained version. For example, in radiative transfer and transport theory [38], the popular Chandrasekhar integral equation, is decretized and presented as (1.1). In signal and image processing problems [36,78], monotone equations are presented as $\ell_{1}$-norm optimization problems. For more on the applications, see $[52,63,86]$. Several iterative schemes for finding solutions of monotone equations exists, and the most popular ones are the Newton and quasi-Newton schemes $[12,17,45,69]$, which possess rapid convergence properties. These methods, however require huge matrix storage at each iterations, which makes them unpopular when engaging problems with large dimensions. As a result, derivative-free methods have been introduced $[1,32,33,50,74,77]$.

Conjugate gradient (CG) method for unconstrained optimization problems is the preferable choice and most appropriate alternative to the aforementioned schemes, when dealing with problems with large dimensions. This is due to the fact that the scheme requires less memory to implement and possess strong convergence properties $[7,49]$. The CG iterative schemes are mostly applied to solve the following minimization problem:

$$
\min _{x \in \mathbf{R}^{n}} \psi(x),
$$

with $\psi: \mathbf{R}^{n} \longrightarrow \mathbf{R}$ representing a real-valued nonlinear mapping, whose gradient is attainable. If the merit function $\psi$ is defined by $\psi:=\left(\frac{1}{2}\right)\|g(x)\|^{2}$, where $\|\cdot\|$ represents the Euclidean norm, then (1.1) is clearly equivalent to the global optimization problem represented in (1.3). The CG method is implemented using the following recursive formula

$$
x_{0} \in \mathbf{R}^{n}, \quad x_{k+1}=x_{k}+t_{k} d_{k}, \quad k \geq 0 .
$$

In (1.4), $x_{k}$ denotes the current iterate, $t_{k}$ a step length obtained using any proper line search procedure, and $d_{k}$ represents the CG search direction given by

$$
\begin{aligned}
d_{0} & =-\nabla \psi\left(x_{0}\right), & k & =0, \\
d_{k} & =-\nabla \psi\left(x_{k}\right)+\beta_{k} d_{k-1}, & & k \geq 1,
\end{aligned}
$$

where $\beta_{k}$ is an essential parameter, which distinguishes the CG schemes [3]. Over the decades, numerous CG algorithms conforming to various types of $\beta_{k}$ in (1.5) were presented (see $[8,9,15,21,51,65,71]$ ). Although these methods often display numerical efficiency (see $[6,10,14,25,26,40]$ ), in most cases the search directions they generate are not descent directions, namely, the condition

$$
\nabla \psi\left(x_{k}\right)^{T} d_{k} \leq-\Phi\left\|\nabla \psi\left(x_{k}\right)\right\|^{2}, \quad \Phi>0, \quad \forall k .
$$

may not be satisfied by the methods. In order to remedy this deficiency, researchers in recent years have engaged more on CG methods with descent directions. By exploiting Perry's approach [56], Liu and Shang [47] developed a CG method with descent search directions, that yield prototypes on which other specified versions of the Perry scheme such as Hestenes-Stiefel [37] method and the Dai and Liao method [14] are developed. In [48], a new Perry CG method, which generates descent search directions irrespective of the line search used, was developed by Liu and Xu. By considering the self-scaling memoryless BFGS update, Andrei [4] proposed an adaptive class of Perry-type CG algorithms with descent search directions that are obtained as a result of symmetrization of the CG direction in [56].

Motivated by the nice properties of CG methods for unconstrained optimization, researchers have proposed their extensions for solving (1.1) and its constrained version. By combining a Polak-Ribieré-Polyak (PRP) scheme $[57,58]$ with the projection method [64], Cheng [13] developed a CG algorithm for solving monotone nonlinear equations, which converges globally to solutions of the given problems. The authors, however indicated 
that global convergence of the method is dependent on its monotonicity and Lipschitz continuous property only. Also, Yu [81] presented a version of the classical PRP [57,58] method for solving nonlinear systems of equations. As with typical CG methods, the scheme is derivative-free and utilizes the line search procedure, modified by Grippo et al. [27] and Li-Fukushima [44]. Furthermore, Dai et al. [16] also proposed a derivative-free method for solving monotone nonlinear equations by combining the modified Perry method [49] with the projection method [64]. The scheme converges globally and is considered as an improvement of the classical Perry method [49]. By employing a modified secant equation and carrying out eigenvalue study of a modified Dai-Liao search direction matrix, Waziri et al. [72] proposed an effective CG method, which converges globally for nonlinear systems of equations. Using a similar approach as in [72], Waziri et al. [70] derived suitable approximations for the unmodified HZ parameter in [29]. The new parameter choices were used to develop effective search directions, which were subsequently combined with the projection scheme [64] to develop effective methods for solving (1.1), which converge globally. Sabi'u et al. [60] improved on the work in [70] by obtaining other choices for the HZ parameter in [29], which were employed to develop other versions of the scheme in [70]. Recently, Sabi'u et al. [61] extended the HZ scheme to solve system of monotone nonlinear equations with convex constraint, by obtaining two other choices for the HZ parameter using singular value approach. For other recent advances in the literature, the reader is referred to $[2,34,35,39,68,71,73,75,76,84]$. For this article, two other adaptations of $\mathrm{HZ}$ method, which are extension of the works in $[60,61,70]$ are presented for solving convex constrained monotone nonlinear equations. This research is embarked upon due to the following considerations:

(i) The HZ method [29] has been well-researched in unconstrained minimization, but its study in constrained monotone nonlinear equations is rare.

(ii) Only few recent study of the HZ scheme for unconstrained as well as constrained monotone system of nonlinear equations considers its applications to real life problems and situations.

Attributes of the new methods include satisfying the condition necessary for global convergence, converging globally to solutions of problems and solving non-smooth problems. Apart from adding to the very few HZ methods that exist for solving systems of monotone nonlinear equations with convex constraints, another contribution of the new schemes is their applications in sparse signal reconstruction and image de-blurring problems.

We organized the remaining sections of the article as follows: Section 2 deals with preliminaries leading to derivation of the methods as well as their algorithms. Analysis of the methods' convergence properties are presented in Section 3 and reports of numerical experiments conducted to highlight effectiveness of the methods proposed are presented in Section 4. Applications of the methods in compressed sensing are presented in Section 5. Section 6 is used to make concluding remarks.

\section{Preliminaries AND the PROposed Methods}

This section presents preliminaries as well as details involved in deriving the methods proposed. Before we proceed to derive the methods, we first describe the projection method [64], for which a sequence $\left\{z_{k}\right\}$ is generated, where

$$
z_{k}=x_{k}+t_{k} d_{k}
$$

and $t_{k}>0$ is a step size obtained by employing appropriate line search method in the direction $d_{k}$ so that

$$
g\left(z_{k}\right)^{T}\left(x_{k}-z_{k}\right)>0
$$

Since $g$ is a monotone mapping, then for each of solution $x^{*}$ of (1.1), we can have

$$
g\left(z_{k}\right)^{T}\left(x^{*}-z_{k}\right)=\left(g\left(z_{k}\right)-g\left(x^{*}\right)\right)^{T}\left(x^{*}-z_{k}\right) \leq 0 .
$$

Hence, by (2.2) and (2.3) we have that the hyperplane

$$
\mathbb{H}_{k}=\left\{x \in \mathbf{R}^{n} \mid g\left(z_{k}\right)^{T}\left(x-z_{k}\right)=0\right\},
$$


separates the current iterate $x_{k}$ strictly from the solution $x^{*}$ of (1.1). In [64], this idea was employed and the authors suggested that the next iterate $x_{k+1}$ should be the projection of $x_{k}$ onto the hyperplane $H_{k}$ and determined as

$$
x_{k+1}=x_{k}-\frac{g\left(z_{k}\right)^{T}\left(x_{k}-z_{k}\right)}{g\left(z_{k}\right)^{T} g\left(z_{k}\right)} g\left(z_{k}\right) .
$$

In [85], Zhang and Zhou presented a new line search with backtracking technique to complete the projection scheme, where the steplength $t_{k}$ is obtained as

$$
t_{k}=\max \left\{\bar{\sigma} \gamma^{i}: i=0,1,2, \ldots\right\},
$$

satisfying

$$
-g\left(x_{k}+t_{k} d_{k}\right)^{T} d_{k} \geq \eta t_{k}\left\|d_{k}\right\|^{2}
$$

with $\gamma \in(0,1), \eta>0$ and $\bar{\sigma} \in(0,1)$ is set as the initial value for the steplength $t_{k}$. Here and in the remaining parts of the article, $\|\cdot\|$ denotes the $\ell_{2}$-norm,$\langle\cdot, \cdot\rangle$ denotes inner product of vectors, $g_{k}=g\left(x_{k}\right), g_{k-1}=g\left(x_{k-1}\right)$, $y_{k-1}=g\left(x_{k}\right)-g\left(x_{k-1}\right), s_{k-1}=x_{k}-x_{k-1}$, and $\Pi_{\Omega}(\cdot)$ stands for the projection operator, which exhibits the nonexpansive property:

$$
\left\|\Pi_{\Omega}(x)-\Pi_{\Omega}(y)\right\| \leq\|x-y\|, \quad \forall x, y \in \mathbf{R}^{n},
$$

and consequently

$$
\left\|\Pi_{\Omega}(x)-y\right\| \leq\|x-y\|, \quad \forall y \in \Omega .
$$

The assumptions stated below are also required in the next section of the paper:

Assumption 2.1. The mapping $g$ is monotone.

Assumption 2.2. A solution $x^{*} \in \Omega$ exists such that $g\left(x^{*}\right)=0$.

Assumption 2.3. The mapping $g$ is Lipschitz continuous; namely, there exists a positive constant $L$ such that for all $x, y \in \boldsymbol{R}^{n}$, the following holds:

$$
\|g(x)-g(y)\| \leq L\|x-y\| .
$$

Hager and Zhang [28] presented a CG scheme that is much related to the method by Perry [55] and Shanno [62]. Particularly, Hager and Zhang $[28,30]$ presented the scheme as

$$
\begin{aligned}
d_{k} & =-\nabla \psi_{k}+\beta_{k}^{N} d_{k-1}, \quad d_{0}=-\nabla \psi_{0}, \\
\beta_{k}^{N} & =\frac{\nabla \psi_{k}^{T} y_{k-1}}{d_{k-1}^{T} y_{k-1}}-2 \frac{\left\|y_{k-1}\right\|^{2} \nabla \psi_{k}^{T} d_{k-1}}{\left(d_{k-1}^{T} y_{k-1}\right)^{2}} .
\end{aligned}
$$

A remarkable feature of the method in (2.11) and (2.12) is that it generates descent directions, i.e., the condition

$$
\nabla \psi_{k}^{T} d_{k} \leq-\frac{7}{8}\left\|\nabla \psi_{k}\right\|^{2}, \quad \forall k \geq 1
$$

is satisfied. In furtherance of (2.11) and (2.12) and to achieve global convergence, Hager and Zhang [28,30] improved on it and gave a modified version with update parameter

$$
\bar{\beta}_{k}^{N}=\max \left\{\beta_{k}^{N}, \bar{\eta}_{k}\right\}, \quad \bar{\eta}_{k}=\frac{-1}{\left\|d_{k}\right\| \min \left\{\bar{\eta},\left\|\nabla \psi_{k}\right\|\right\}},
$$

were $\bar{\eta}$ is a positive constant. Furthermore, by making some modifications in the classical Hestenes-Stiefel (HS) [37] scheme, Hager and Zhang [29] proposed the following one-parameter CG update:

$$
\beta_{k}^{\theta}=\frac{\nabla \psi_{k}^{T} y_{k-1}}{d_{k-1}^{T} y_{k-1}}-\theta_{k} \frac{\left\|y_{k-1}\right\|^{2}}{d_{k-1}^{T} y_{k-1}} \frac{\nabla \psi_{k}^{T} d_{k-1}}{d_{k-1}^{T} y_{k-1}}, \quad\left(\theta_{k} \geq 0\right) .
$$


It can be observed that for $\theta_{k}=2,(2.15)$ reduces to the scheme in (2.12). Considering that the parameter $\theta_{k}$ is nonnegative, researchers in recent years have shown interests in finding appropriate values for the parameter. By conducting eigenvalue study Waziri et al. [70] modified the HZ scheme (2.15) and gave the following approximations for the parameter $\theta_{k}$ :

$$
\theta_{k 1}=R 1-R 2 \frac{\left(s_{k-1}^{T} y_{k-1}\right)^{2}}{\left\|y_{k-1}\right\|^{2}\left\|s_{k-1}\right\|^{2}}
$$

where $R 1>\frac{1}{4}$ and $R 2 \leq 0$, and

$$
\theta_{k 2}=R 3-R 4 \frac{\left(s_{k-1}^{T} \bar{z}_{k-1}\right)^{2}}{\left\|\bar{z}_{k-1}\right\|^{2}\left\|s_{k-1}\right\|^{2}}
$$

where

$$
\bar{z}_{k-1}=y_{k-1}+\hat{\zeta} \frac{\max \left\{v_{k}, 0\right\}}{s_{k-1}^{T} d_{k-1}} d_{k-1}, \quad v_{k}=6\left(\psi_{k-1}-\psi_{k}\right)+3\left\langle g_{k-1}+g_{k}, s_{k-1}\right\rangle, \quad \hat{\zeta}>0
$$

with $R 3>\frac{1}{4}$ and $R 4 \leq 0$. Clearly, in both (2.16) and (2.17) $\theta_{k}>\frac{1}{4}$, for which the authors showed that the scheme in [70] satisfies the inequality necessary for global convergence, namely

$$
g_{k}^{T} d_{k} \leq-\Gamma\left\|g_{k}\right\|^{2}, \quad \forall k \geq 1
$$

By minimizing the Frobenius condition number of a search direction matrix as well as minimizing the difference between the smallest and largest singular value, Sabi'u et al. [60] presented two HZ-type schemes with the following approximations of the HZ parameter:

$$
\theta_{k 1}^{*}=\frac{\left(s_{k-1}^{T} \bar{w}_{k-1}\right)^{2}}{\left\|s_{k-1}\right\|^{2}\left\|\bar{w}_{k-1}\right\|^{2}}
$$

and

where

$$
\theta_{k 2}^{*}=\sqrt{\frac{\left\|s_{k-1}\right\|\left\|\bar{w}_{k-1}\right\|}{s_{k-1}^{T} \bar{w}_{k-1}}}
$$

$$
\bar{w}_{k-1}=y_{k-1}+\bar{\rho} \frac{\max \left\{v_{k}, 0\right\}}{\left\|s_{k-1}\right\|^{2}} s_{k-1}, \quad \bar{\rho} \in[0,1] .
$$

The authors proved that the methods are effective and converge globally under mild conditions. Recently, inspired by the work in $[8,60,70]$, Sabi'u et al. [61] proposed two other HZ-type methods for convex constrained monotone equations with the following parameters:

$$
\theta_{k 3}^{*}=1+\sqrt{1+\left(\frac{s_{k-1}^{T} \bar{w}_{k-1}}{\left\|s_{k-1}\right\|\left\|\bar{w}_{k-1}\right\|}\right)^{2}}
$$

and

$$
\theta_{k 4}^{*}=\frac{\left\|s_{k-1}\right\|^{2}\left\|\bar{w}_{k-1}\right\|^{2}}{\left(s_{k-1}^{T} \bar{w}_{k-1}\right)^{2}}+\sqrt{\frac{\left(s_{k-1}^{T} \bar{w}_{k-1}\right)^{2}}{\left\|s_{k-1}\right\|^{2}\left\|\bar{w}_{k-1}\right\|^{2}}+\frac{\left\|s_{k-1}\right\|^{4}\left\|\bar{w}_{k-1}\right\|^{4}}{\left(s_{k-1}^{T} \bar{w}_{k-1}\right)^{4}}},
$$

where

$$
\bar{w}_{k-1}=y_{k-1}+\bar{\rho} \frac{\max \left\{v_{k}, 0\right\}}{\left\|s_{k-1}\right\|^{2}} s_{k-1}, \quad \bar{\rho} \in[0,1] .
$$

Still, finding optimal values for the parameter $\theta_{k}$ of the one-parameter HZ method is open for research. So, with inspiration from the works in $[60,61,70]$, we also propose two optimal choices, for the HZ parameter, and consequently present two modified HZ methods for system of nonlinear monotone equations with convex constraint, which are effective and always satisfy the condition (2.19). 


\subsection{First proposed method}

By considering (2.15), we suggest a modified HZ search direction defined by

$$
d_{k}=-g_{k}+\left(\frac{g_{k}^{T} \tilde{y}_{k-1}}{s_{k-1}^{T} \tilde{y}_{k-1}}-\theta_{k} \frac{\left\|\tilde{y}_{k-1}\right\|^{2}}{\tilde{y}_{k-1}^{T} s_{k-1}} \frac{g_{k}^{T} s_{k-1}}{\tilde{y}_{k-1}^{T} s_{k-1}}\right) s_{k-1},
$$

where $\tilde{y}_{k-1}=y_{k-1}+c s_{k-1}$, with $c \in \mathbf{R}, \quad c>0$.

Proposition 2.4. For all $k \geq 0$, the search direction presented in (2.26) is well-defined.

Proof. For (2.26) to be well-defined, $\tilde{y}_{k-1}^{T} s_{k-1}$ must not be equal to zero. Using the definition of $y_{k-1}$, we have

$$
\tilde{y}_{k-1}^{T} s_{k-1}=\left(y_{k-1}+c s_{k-1}\right)^{T} s_{k-1}=\left(g\left(x_{k}\right)-g\left(x_{k-1}\right)\right)^{T}\left(x_{k}-x_{k-1}\right)+c\left\|s_{k-1}\right\|^{2},
$$

and applying the monotonicity of the mapping $g$, yields

$$
\tilde{y}_{k-1}^{T} s_{k-1} \geq c\left\|s_{k-1}\right\|^{2}>0 .
$$

Also, equation (2.26) can be re-written as

$$
d_{k}=-E_{k} g_{k}
$$

where $E_{k}$ in $(2.29)$ is a search direction matrix defined by

$$
E_{k}=I-\frac{s_{k-1} \tilde{y}_{k-1}^{T}}{\tilde{y}_{k-1}^{T} s_{k-1}}+\theta_{k} \frac{\left\|\tilde{y}_{k-1}\right\|^{2}}{\tilde{y}_{k-1}^{T} s_{k-1}} \frac{s_{k-1} s_{k-1}^{T}}{\tilde{y}_{k-1}^{T} s_{k-1}} .
$$

Interestingly, equation (2.30) is similar to the quasi-Newton scheme in [65], whose updating scheme is defined as

$$
H_{k}=I-\frac{s_{k-1} \tilde{y}_{k-1}^{T}+y_{k-1} s_{k-1}^{T}}{\tilde{y}_{k-1}^{T} s_{k-1}}+\left(1+\frac{\left\|y_{k-1}\right\|^{2}}{\tilde{y}_{k-1}^{T} s_{k-1}}\right) \frac{s_{k-1} s_{k-1}^{T}}{\tilde{y}_{k-1}^{T} s_{k-1}},
$$

where $H_{k}$ approximates the inverse Hessian $\nabla^{2} \psi\left(x_{k}\right)^{-1}$. Since $E_{k}$ is a rank-two update, with the formula in [65], namely

$$
\operatorname{det}\left(I+a_{1} a_{2}^{T}+a_{3} a_{4}^{T}\right)=\left(1+a_{1}^{T} a_{2}\right)\left(1+a_{3}^{T} a_{4}\right)-\left(a_{1}^{T} a_{4}\right)\left(a_{2}^{T} a_{3}\right),
$$

we obtain its determinant as

$$
\operatorname{det}\left(E_{k}\right)=\theta_{k} \frac{\left\|\tilde{y}_{k-1}\right\|^{2}\left\|s_{k-1}\right\|^{2}}{\left(\tilde{y}_{k-1}^{T} s_{k-1}\right)^{2}} .
$$

It can be observed that the matrix in (2.31) is symmetric but the matrix $E_{k}$ in (2.30) is not. So, to obtain a similar form for (2.30) as (2.31), we need to symmetrize $E_{k}$. Using Perry's approach [56] and employing a rank-one update, we obtain the following version of (2.30):

$$
A_{k}=I-\frac{s_{k-1} \tilde{y}_{k-1}^{T}+\tilde{y}_{k-1} s_{k-1}^{T}}{\tilde{y}_{k-1}^{T} s_{k-1}}+\theta_{k} \frac{\left\|\tilde{y}_{k-1}\right\|^{2}}{\tilde{y}_{k-1}^{T} s_{k-1}} \frac{s_{k-1} s_{k-1}^{T}}{\tilde{y}_{k-1}^{T} s_{k-1}} .
$$

It is observed that if we take

$$
\theta_{k}=1+\frac{\tilde{y}_{k-1}^{T} s_{k-1}}{\left\|\tilde{y}_{k-1}\right\|^{2}}
$$


then $A_{k}$ reduces to the memoryless BFGS method discussed in [5] and defined by (2.31). Using (2.34), we present the revised search direction as

$$
d_{k}=-A_{k} g_{k}, \quad 0,1, \ldots
$$

Now, in order to obtain another appropriate choice for the parameter $\theta_{k}$ that will consequently guarantee the condition (2.19) as well as conditioning of the symmetric matrix $A_{k}$, we conduct eigenvalue analysis of $A_{k}$.

Theorem 2.5. Let the matrix $A_{k}$ be given by (2.34) with $\theta_{k}>1, \forall k$. Then the eigenvalues of $A_{k}$ are 1 , with multiplicity $(n-2), \xi_{k}^{+}$and $\xi_{k}^{-}$. Also, the last two eigenvalues $\xi_{k}^{+}$and $\xi_{k}^{-}$are positive real numbers.

Proof. By (2.28) $\tilde{y}_{k-1}^{T} s_{k-1}>0$, therefore, each vectors $\tilde{y}_{k-1}$ and $s_{k-1}$ are nonzero. Let $\varsigma$ be the vector space spanned by $\tilde{y}_{k-1}$ and $s_{k-1}$, namely, $\varsigma=\operatorname{span}\left\{\tilde{y}_{k-1}, s_{k-1}\right\} \subset \mathbf{R}^{n}$, then $\operatorname{dim}(\varsigma) \leq 2$. Also, let $\varsigma^{\perp}$ be the orthogonal complement of $\varsigma$, then $\operatorname{dim}\left(\varsigma^{\perp}\right) \geq n-2$. Therefore, a set of mutually orthogonal vectors $\left\{\nu_{k-1}^{i}\right\}_{i=1}^{n-2} \subset \varsigma^{\perp}$ exists such that

which from (2.34) leads to

$$
\tilde{y}_{k-1}^{T} \nu_{k-1}^{i}=s_{k-1}^{T} \nu_{k-1}^{i}=0,
$$

$$
A_{k} \nu_{k-1}^{i}=A_{k}^{T} \nu_{k-1}^{i}=\nu_{k-1}^{i}, \quad i=1, \ldots, n-2 .
$$

So, for $i=1, \ldots, n-2, \nu_{k-1}^{i}$, represents eigenvectors of the matrix $A_{k}$ corresponding to eigenvalue 1 each. Let the other two eigenvalues of $A_{k}$ be $\xi_{k}^{+}$and $\xi_{k}^{-}$respectively. From (2.34), we can re-write $A_{k}$ as

$$
A_{k}=I-\frac{s_{k-1}\left(\left(\tilde{y}_{k-1}^{T} s_{k-1}\right) \tilde{y}_{k-1}-\theta_{k}\left\|\tilde{y}_{k-1}\right\|^{2} s_{k-1}\right)^{T}}{\left(\tilde{y}_{k-1}^{T} s_{k-1}\right)^{2}}-\frac{\tilde{y}_{k-1} s_{k-1}^{T}}{\tilde{y}_{k-1}^{T} s_{k-1}},
$$

which clearly represents a rank-two update. So, using similar approach as in the previous case, we obtain the determinant as

$$
\operatorname{det}\left(A_{k}\right)=\theta_{k} \frac{\left\|s_{k-1}\right\|^{2}\left\|\tilde{y}_{k-1}\right\|^{2}}{\tilde{y}_{k-1}^{T} s_{k-1}}-\frac{\left\|s_{k-1}\right\|^{2}\left\|\tilde{y}_{k-1}\right\|^{2}}{\tilde{y}_{k-1}^{T} s_{k-1}} .
$$

Also, from (2.34) and the fact that for a square symmetric matrix, the trace is equivalent to sum of its eigenvalues, we obtain

$$
\operatorname{trace}\left(A_{k}\right)=n-2+\theta_{k} \frac{\left\|s_{k-1}\right\|^{2}}{\tilde{y}_{k-1}^{T} s_{k-1}} \frac{\left\|\tilde{y}_{k-1}\right\|^{2}}{\tilde{y}_{k-1}^{T} s_{k-1}}=\underbrace{1+\ldots+1}_{(n-2) \text { times }}+\xi_{k}^{+}+\xi_{k}^{-},
$$

for which we have

$$
\xi_{k}^{+}+\xi_{k}^{-}=\theta_{k} \frac{\left\|s_{k-1}\right\|^{2}\left\|\tilde{y}_{k-1}\right\|^{2}}{\left(\tilde{y}_{k-1}^{T} s_{k-1}\right)^{2}} .
$$

From (2.42), we see that for $\theta_{k}>0$,

$$
\xi_{k}^{+}+\xi_{k}^{-}>0
$$

Therefore, for the matrix $A_{k}$ to be positive-definite, we require

$$
\xi_{k}^{+} \xi_{k}^{-}=\theta_{k} \frac{\left\|s_{k-1}\right\|^{2}\left\|\tilde{y}_{k-1}\right\|^{2}}{\tilde{y}_{k-1}^{T} s_{k-1}}-\frac{\left\|s_{k-1}\right\|^{2}\left\|\tilde{y}_{k-1}\right\|^{2}}{\tilde{y}_{k-1}^{T} s_{k-1}}>0 .
$$

Utilizing (2.40) we see that (2.44) is satisfied if

$$
\theta_{k}>1
$$

Now, for simplicity, we set

$$
u_{k}=\frac{\left\|s_{k-1}\right\|^{2}\left\|\tilde{y}_{k-1}\right\|^{2}}{\left(\tilde{y}_{k-1}^{T} s_{k-1}\right)^{2}} .
$$


Hence, by utilizing (2.40) and (2.42), $\xi_{k}^{+}$and $\xi_{k}^{-}$are obtained as the roots of the quadratic equation:

$$
\xi^{2}-\theta_{k} u_{k} \xi+\theta_{k} u_{k}-u_{k}=0
$$

Specifically, we can write

$$
\xi_{k}^{ \pm}=\frac{1}{2}\left[\theta_{k} u_{k} \pm \sqrt{\left(\theta_{k} u_{k}-2\right)^{2}+4\left(\theta_{k} u_{k}-1\right)}\right] .
$$

Utilizing (2.45), (2.46), the Cauchy-Schwartz inequality and performing some algebra, we have that

$$
0<\xi_{k}^{-}<1<\xi_{k}^{+} \text {. }
$$

Now, multiplying through $(2.39)$ by $g_{k}$ and setting $\xi_{k}^{-}=\xi \in(0,1)$, we have

$$
d_{k}^{T} g_{k}=-g_{k}^{T} A_{k} g_{k} \leq-\xi\left\|g_{k}\right\|^{2}<0,
$$

for which the positive definiteness of $A_{k}$ is satisfied.

Definition 2.6 ([67]). Given any arbitrary nonsingular matrix $\bar{H}$, the condition number of $\bar{H}$ is a scalar $\kappa(\bar{H})$, which is defined by

$$
\kappa(\bar{H})=\|\bar{H}\|\left\|\bar{H}^{-1}\right\|
$$

In [67], it was noted that decreasing the condition number can improve the numerical stability of a matrixbased computational process. Hence, an optimal choice for $\theta_{k}$ for which the condition number of $A_{k}$ is minimized, is determined by minimizing the distance between the eigenvalues $\xi_{k}^{+}$and $\xi_{k}^{-}$and making them as close as possible. Namely, the optimal value of $\theta_{k}$ is given by

$$
\bar{\theta}_{k 1}=\arg \min _{\theta_{k}>0}\left(\xi_{k}^{+}-\xi_{k}^{-}\right)=2 \frac{\left(\tilde{y}_{k-1}^{T} s_{k-1}\right)^{2}}{\left\|s_{k-1}\right\|^{2}\left\|\tilde{y}_{k-1}\right\|^{2}} .
$$

Still, equation (2.52) as defined may fail at some instances to satisfy (2.45), in which case $A_{k}$ cannot be positivedefinite. So, to guard against such situations, we propose the following choice for the parameter $\theta_{k}$ :

$$
\theta_{k 1}^{*}=\max \left\{\bar{\theta}_{k 1}, \vartheta\right\}, \quad \vartheta \in(1, \infty) .
$$

We therefore, write the revised modified HZ update parameter as

$$
\beta_{k}^{\theta_{k 1}^{*}}=\frac{g_{k}^{T} \tilde{y}_{k-1}}{s_{k-1}^{T} \tilde{y}_{k-1}}-\theta_{k 1}^{*} \frac{\left\|\tilde{y}_{k-1}\right\|^{2}}{s_{k-1}^{T} \tilde{y}_{k-1}} \frac{g_{k}^{T} s_{k-1}}{s_{k-1}^{T} \tilde{y}_{k-1}},
$$

with search direction given by

$$
\begin{array}{rlrl}
d_{0}^{\theta_{k 1}^{*}}=-g_{0}, & k & =0, \\
d_{k}^{\theta_{k 1}^{*}}=-g_{k}+\beta_{k}^{\theta_{k 1}^{*}} s_{k-1}, & k=1,2, \ldots,
\end{array}
$$

where $\beta_{k}^{\theta_{k 1}^{*}}$ is as defined in (2.54).

Lemma 2.7. The search direction defined by (2.54) and (2.55) satisfies the inequality,

$$
g_{k}^{T} d_{k} \leq-\Phi\left\|g_{k}\right\|^{2}, \quad k=0,1,2, \ldots,
$$

where $\Phi=\left(1-\frac{1}{4 \theta_{k 1}^{*}}\right)$ and $\theta_{k 1}^{*}>\frac{1}{4}$. 
Proof. For $k=0$, using (2.55) we have $g_{0}^{T} d_{0}=\left\|g_{0}\right\|^{2}$, where $\Phi=1$. Now, for $k \geq 1$, we consider two cases.

Case 1. $\vartheta \geq \bar{\theta}_{k 1}$, then $\theta_{k 1}^{*}=\vartheta$. So, using (2.54) and (2.55), we have

$$
\begin{aligned}
g_{k}^{T} d_{k}^{\theta_{k 1}^{*}} & =-\left\|g_{k}\right\|^{2}+\frac{g_{k}^{T} \tilde{y}_{k-1}}{s_{k-1}^{T} \tilde{y}_{k-1}} g_{k}^{T} s_{k-1}-\frac{\vartheta\left\|\tilde{y}_{k-1}\right\|^{2}\left(g_{k}^{T} s_{k-1}\right)^{2}}{\left(s_{k-1}^{T} \tilde{y}_{k-1}\right)^{2}} \\
& =\frac{g_{k}^{T} \tilde{y}_{k-1}\left(s_{k-1}^{T} \tilde{y}_{k-1}\right)\left(g_{k}^{T} s_{k-1}\right)-\left\|g_{k}\right\|^{2}\left(s_{k-1}^{T} \tilde{y}_{k-1}\right)^{2}-\vartheta\left\|\tilde{y}_{k-1}\right\|^{2}\left(g_{k}^{T} s_{k-1}\right)^{2}}{\left(s_{k-1}^{T} \tilde{y}_{k-1}\right)^{2}} .
\end{aligned}
$$

By setting $u_{1}=\frac{1}{\sqrt{2 \vartheta}}\left(s_{k-1}^{T} \tilde{y}_{k-1}\right) g_{k}, \quad u_{2}=\sqrt{2 \vartheta}\left(g_{k}^{T} s_{k-1}\right) \tilde{y}_{k-1}$ and applying the inequality,

$$
u_{1}^{T} u_{2} \leq \frac{1}{2}\left(\left\|u_{1}\right\|^{2}+\left\|u_{2}\right\|^{2}\right)
$$

on the first term of the second equality in (2.57), we obtain

$$
\begin{aligned}
g_{k}^{T} d_{k}^{\theta_{k 1}^{*}} & \leq \frac{-\left(1-\frac{1}{4 \vartheta}\right)\left(s_{k-1}^{T} \tilde{y}_{k-1}\right)^{2}\left\|g_{k}\right\|^{2}+\vartheta\left\|\tilde{y}_{k-1}\right\|^{2}\left(g_{k}^{T} s_{k-1}\right)^{2}-\vartheta\left\|\tilde{y}_{k-1}\right\|^{2}\left(g_{k}^{T} s_{k-1}\right)^{2}}{\left(s_{k-1}, \tilde{y}_{k-1}\right)^{2}} \\
& =-\left(1-\frac{1}{4 \vartheta}\right)\left\|g_{k}\right\|^{2} .
\end{aligned}
$$

Case 2. $\vartheta<\bar{\theta}_{k 1}$, then $\theta_{k 1}^{*}=\bar{\theta}_{k 1}$, where $\bar{\theta}_{k 1}$ is as defined in (2.52). So, using (2.54) and (2.55), we have

$$
\begin{aligned}
g_{k}^{T} d_{k}^{\theta_{k 1}^{*}} & =-\left\|g_{k}\right\|^{2}+\frac{g_{k}^{T} \tilde{y}_{k-1}}{s_{k-1}^{T} \tilde{y}_{k-1}} g_{k}^{T} s_{k-1}-\frac{\bar{\theta}_{k 1}\left\|\tilde{y}_{k-1}\right\|^{2}\left(g_{k}^{T} s_{k-1}\right)^{2}}{\left(s_{k-1}^{T} \tilde{y}_{k-1}\right)^{2}} \\
& =\frac{g_{k}^{T} \tilde{y}_{k-1}\left(s_{k-1}^{T} \tilde{y}_{k-1}\right)\left(g_{k}^{T} s_{k-1}\right)-\left\|g_{k}\right\|^{2}\left(s_{k-1}^{T} \tilde{y}_{k-1}\right)^{2}-\bar{\theta}_{k 1}\left\|\tilde{y}_{k-1}\right\|^{2}\left(g_{k}^{T} s_{k-1}\right)^{2}}{\left(s_{k-1}^{T} \tilde{y}_{k-1}\right)^{2}} .
\end{aligned}
$$

Applying similar argument as in case 1, we obtain

$$
\begin{aligned}
g_{k}^{T} d_{k}^{\theta_{k 1}^{*}} & \leq \frac{-\left(1-\frac{1}{4 \bar{\theta}_{k 1}}\right)\left(s_{k-1}^{T} \tilde{y}_{k-1}\right)^{2}\left\|g_{k}\right\|^{2}+\bar{\theta}_{k 1}\left\|\tilde{y}_{k-1}\right\|^{2}\left(g_{k}, s_{k-1}\right)^{2}-\bar{\theta}_{k 1}\left\|\tilde{y}_{k-1}\right\|^{2}\left(g_{k}^{T} s_{k-1}\right)^{2}}{\left(s_{k-1}^{T} \tilde{y}_{k-1}\right)^{2}} \\
& =-\left(1-\frac{1}{4 \bar{\theta}_{k 1}}\right)\left\|g_{k}\right\|^{2} .
\end{aligned}
$$

So, if $\bar{\theta}_{k 1}=\bar{\theta}>\frac{1}{4}$, the result is established. Consequently, in both cases, equation (2.56) is satisfied. 
The algorithm for the first scheme is described as follows:

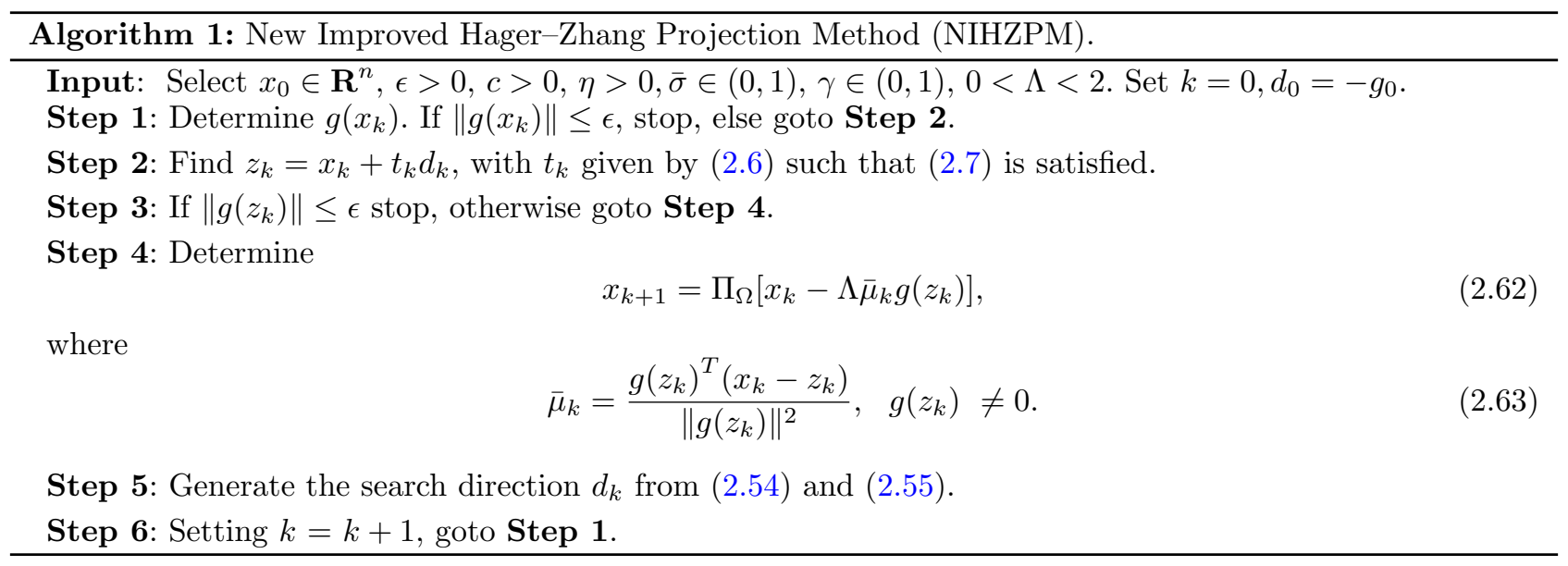

\subsection{The second proposed method}

Here, we conduct singular value study of the non-singular nonsymmetric matrix $E_{k}$ in (2.30) and consequently suggest another optimal value for the HZ parameter. First, using (2.35), we propose the following adaptive choice for $\theta_{k}$ :

$$
\theta_{k 2}=\varphi\left(\frac{s_{k-1}^{T} \tilde{y}_{k-1}}{\left\|\tilde{y}_{k-1}\right\|^{2}}+1\right),
$$

where $\varphi>0$ represents a real parameter. It can be observed that setting $\varphi=1, E_{k}$ becomes similar to the Memoryless BFGS updating formula presented in (2.31). Next, we give the following definition:

Definition $2.8([67])$. Suppose $Q \in \mathbf{R}^{n \times n}$ is a non-singular matrix having singular values $\tau_{1} \geq \tau_{2} \geq \ldots \geq \tau_{n}>$ 0 . Then the scalar $\kappa(Q)$ is called its condition number and is defined as

$$
\kappa(Q)=\frac{\tau_{1}}{\tau_{n}} .
$$

To proceed with our discussion, we need to determine singular values of $E_{k}$. Since from $(2.28), s_{k-1}^{T} \tilde{y}_{k-1} \neq 0$, it implies that a set of mutually orthonormal vectors $\left\{\alpha_{k-1}^{i}\right\}_{i=1}^{n-2}$ exists such that

$$
s_{k-1}^{T} \alpha_{k-1}^{i}=\tilde{y}_{k-1}^{T} \alpha_{k-1}^{i}=0, \quad i=1,2, \ldots, n-2,
$$

for which from (2.30), we obtain

$$
E_{k} \alpha_{k-1}^{i}=E_{k}^{T} \alpha_{k-1}^{i}=\alpha_{k-1}^{i}, \quad i=1,2, \ldots, n-2 .
$$

This implies that the matrix $E_{k}$ has $(n-2)$ singular values with multiplicity 1 . Let the remaining two singular values be $\tau_{k}^{+}$and $\tau_{k}^{-}$respectively. By applying properties of Frobenius norm, we have

$$
\left\|E_{k}\right\|_{F}=\sqrt{\operatorname{trace}\left(E_{k}^{T} E_{k}\right)}
$$

Squaring both sides of (2.68), we get

$$
\begin{aligned}
\left\|E_{k}\right\|_{F}^{2} & =\operatorname{trace}\left(E_{k}^{T} E_{k}\right) \\
& =n-2+\frac{\left\|s_{k-1}\right\|^{2}\left\|\tilde{y}_{k-1}\right\|^{2}}{\left(s_{k-1}^{T} \tilde{y}_{k-1}\right)^{2}}+\theta_{k}^{2} \frac{\left\|s_{k-1}\right\|^{4}\left\|\tilde{y}_{k-1}\right\|^{4}}{\left(s_{k-1}^{T} \tilde{y}_{k-1}\right)^{4}} .
\end{aligned}
$$


Also, given an arbitrary matrix $Q \in \mathbf{R}^{n \times m}$ with $\operatorname{rank} r$, we know that

$$
\left\|Q_{k}\right\|_{F}^{2}=\sum_{i=1}^{r} \tau_{i}^{2}
$$

where $\|\cdot\|_{F}$ denotes the Frobenius norm. Hence, using (2.69) and (2.70) we can have

$$
\left(\tau_{k}^{-}\right)^{2}+\left(\tau_{k}^{+}\right)^{2}=\frac{\left\|s_{k-1}\right\|^{2}\left\|\tilde{y}_{k-1}\right\|^{2}}{\left(s_{k-1}^{T} \tilde{y}_{k-1}\right)^{2}}+\theta_{k}^{2} \frac{\left\|s_{k-1}\right\|^{4}\left\|\tilde{y}_{k-1}\right\|^{4}}{\left(s_{k-1}^{T} \tilde{y}_{k-1}\right)^{4}} .
$$

Also, when $r=m=n$, we have

$$
\left|\operatorname{det}\left(Q_{k}\right)\right|=\prod_{i=1}^{n} \tau_{i}
$$

So, by utilizing (2.33) and (2.72), we obtain

$$
\tau_{k}^{-} \tau_{k}^{+}=\theta_{k} \frac{\left\|s_{k-1}\right\|^{2}\left\|\tilde{y}_{k-1}\right\|^{2}}{\left(s_{k-1}^{T} \tilde{y}_{k-1}\right)^{2}} .
$$

Next, we define

$$
w_{k}=\frac{\left\|s_{k-1}\right\|^{2}\left\|\tilde{y}_{k-1}\right\|^{2}}{\left(s_{k-1}^{T} \tilde{y}_{k-1}\right)^{2}} .
$$

Therefore, using (2.33), (2.73), (2.74) with some algebra and rearrangements, we obtain the remaining singular values of $E_{k}$ as

$$
\tau_{k}^{ \pm}=\frac{1}{2}\left[\sqrt{\left(\theta_{k} w_{k}+1\right)^{2}+w_{k}-1} \pm \sqrt{\left(\theta_{k} w_{k}-1\right)^{2}+w_{k}-1}\right] .
$$

Applying the Cauchy-Schwartz inequality and the fact that the parameter $\theta_{k} \geq 0$, we obtain that the singular value $\tau_{k}^{+} \geq 1$. Using similar argument and the fact that $\tau_{k}^{-} \leq \tau_{k}^{+}$, it is easily seen that $\tau_{k}^{-} \leq 1$, and we conclude that $\tau_{k}^{-} \leq 1 \leq \tau_{k}^{+}$. Therefore, we obtain an upper bound for the spectral condition number of $E_{k}$ as follows:

$$
\kappa\left(E_{k}\right)=\frac{\tau_{k}^{+}}{\tau_{k}^{-}} \leq \frac{\left(\tau_{k}^{+}\right)^{2}+\left(\tau_{k}^{-}\right)^{2}}{\tau_{k}^{+} \tau_{k}^{-}} .
$$

Utilizing (2.64), (2.33), and (2.73), we get

$$
\tau_{k}^{+} \tau_{k}^{-}=\varphi\left(\frac{s_{k-1}^{T} \tilde{y}_{k-1}}{\left\|\tilde{y}_{k-1}\right\|^{2}}+1\right) \frac{\left\|s_{k-1}\right\|^{2}\left\|\tilde{y}_{k-1}\right\|^{2}}{\left(s_{k-1}^{T} \tilde{y}_{k-1}\right)^{2}}
$$

and

$$
\left(\tau_{k}^{+}\right)^{2}+\left(\tau_{k}^{-}\right)^{2}=\frac{\left\|s_{k-1}\right\|^{2}\left\|\tilde{y}_{k-1}\right\|^{2}}{\left(s_{k-1}^{T} \tilde{y}_{k-1}\right)^{2}}+\varphi^{2}\left(\frac{s_{k-1}^{T} \tilde{y}_{k-1}}{\left\|\tilde{y}_{k-1}\right\|^{2}}+1\right)^{2} \frac{\left\|s_{k-1}\right\|^{4}\left\|\tilde{y}_{k-1}\right\|^{4}}{\left(s_{k-1}^{T} \tilde{y}_{k-1}\right)^{4}} .
$$

From (2.76), (2.77), and (2.78), we have

$$
\kappa\left(E_{k}\right) \leq \frac{1}{\varphi} \frac{\left\|\tilde{y}_{k-1}\right\|^{2}}{\left(s_{k-1}^{T} \tilde{y}_{k-1}+\left\|\tilde{y}_{k-1}\right\|^{2}\right)}+\varphi\left(\frac{\left\|s_{k-1}\right\|^{2}}{s_{k-1}^{T} \tilde{y}_{k-1}}+\frac{\left\|s_{k-1}\right\|^{2}\left\|\tilde{y}_{k-1}\right\|^{2}}{\left(s_{k-1}^{T} \tilde{y}_{k-1}\right)^{2}}\right) .
$$


After some algebra, we obtain the following as minimizer of the upper bound of the spectral condition number given in (2.79):

$$
\varphi_{k}=\frac{\left\|\tilde{y}_{k-1}\right\| s_{k-1}^{T} \tilde{y}_{k-1}}{\left\|s_{k-1}\right\|\left(s_{k-1}^{T} \tilde{y}_{k-1}+\left\|\tilde{y}_{k-1}\right\|^{2}\right)} .
$$

Therefore, from (2.64) and (2.80), we obtain the value for the HZ parameter as

$$
\bar{\theta}_{k 2}=\frac{s_{k-1}^{T} \tilde{y}_{k-1}}{\left\|s_{k-1}\right\|\left\|\tilde{y}_{k-1}\right\|} .
$$

But, equation (2.81) as defined may not satisfy $(2.56)$ in the interval $\left(0, \frac{1}{4}\right)$. Using earlier approach, the following revised form is proposed:

$$
\theta_{k 2}^{*}=\max \left\{\bar{\theta}_{k 2}, \sigma\right\}, \quad \sigma \in\left(\frac{1}{4}, \infty\right) .
$$

We, therefore, obtain the second revised HZ update parameter as

$$
\beta_{k}^{\theta_{k 2}^{*}}=\frac{g_{k}^{T} \tilde{y}_{k-1}}{s_{k-1}^{T} \tilde{y}_{k-1}}-\theta_{k 2}^{*} \frac{\left\|\tilde{y}_{k-1}\right\|^{2}}{s_{k-1}^{T} \tilde{y}_{k-1}} \frac{g_{k}^{T} s_{k-1}}{s_{k-1}^{T} \tilde{y}_{k-1}},
$$

with search direction given by

$$
\begin{array}{ll}
d_{02}^{\theta_{k 2}^{*}}=-g_{0}, & k=0, \\
d_{k}^{\theta_{k 2}^{*}}=-g_{k}+\beta_{k}^{\theta_{2} *} s_{k-1}, & k=1,2, \ldots,
\end{array}
$$

where $\beta_{k}^{\theta_{2} *}$ is as defined in (2.83).

Lemma 2.9. The search direction given in (2.83) and (2.84) satisfies the inequality (2.56).

Using similar argument as Lemma 2.7, the result can easily be established. Now, following the above discussion, it is imperative to give the following remark regarding the two choices of the HZ parameter obtained, i.e., $\theta_{k 1}^{*}$ and $\theta_{k 2}^{*}$.

Remark 2.10. Careful study of the two choices of modified Hager-Zhang parameters provided in (2.53) and (2.82) indicates that they satisfy the inequality $(2.56)$ for values in the intervals $(1, \infty)$ and $\left(\frac{1}{4}, \infty\right)$. This clearly shows an improvement over the parameter choices for the schemes in [61] given by (2.23) and (2.25), both of which lie in the interval $(2, \infty)$.

Next, we describe the algorithm as follows:

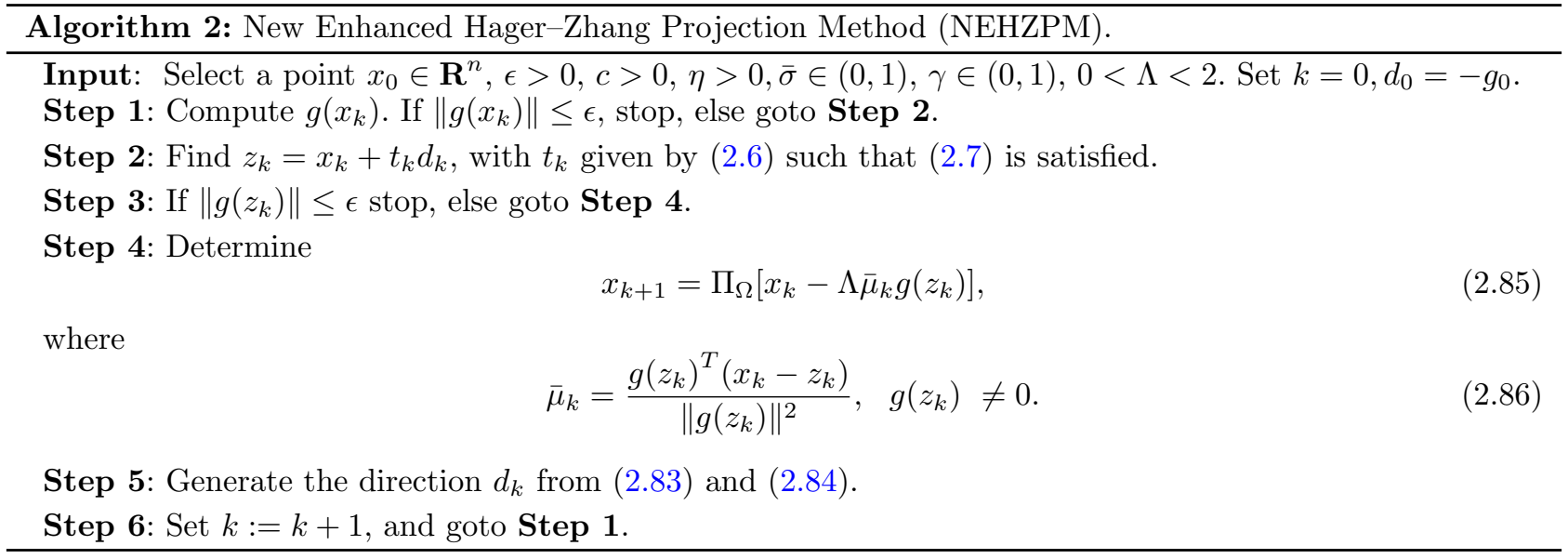


Lemma 2.11. The line search technique (2.7) employed in step 2 of Algorithms 1 and 2 is well-defined.

Proof. We approach the proof using contradiction. Let there exists an iterate index $\bar{k}$ for which (2.7) is not satisfied. Then, by setting $t_{\bar{k}}=\zeta \gamma^{i}$, we have

$$
-g\left(x_{\bar{k}}+\zeta \gamma^{i} d_{\bar{k}}\right)^{T} d_{\bar{k}}<\hat{\sigma} \zeta \gamma^{i}\left\|d_{\bar{k}}\right\|^{2}, i \geq 0 .
$$

By employing continuity of the function $g$ and allowing the nonnegative integer $i$ to approach infinity in (2.87), i.e., $i \rightarrow \infty$, we have

$$
g\left(x_{\bar{k}}\right)^{T} d_{\bar{k}} \leq 0 .
$$

From (2.56) and (2.88), it is easy to deduce that $g\left(x_{\bar{k}}\right)=0$. Interestingly, for both Algorithms 1 and 2, considering steps 1 and 2 , when the line search is calculated, indicates that $g\left(x_{\bar{k}}\right) \neq 0$, and this clearly contradicts the result that $g\left(x_{\bar{k}}\right)=0$.

\section{Convergence Results of the methods}

Here and in the rest of this section, global convergence of Algorithms 1 and 2 is discussed. We proceed with the following lemma, which holds for both algorithms.

Lemma 3.1. Given that Assumptions 2.1-2.3 hold, then the sequences $\left\{x_{k}\right\}$ and $\left\{z_{k}\right\}$ generated by Algorithm 1 are bounded and

$$
\lim _{k \rightarrow \infty} t_{k}\left\|d_{k}\right\|=0
$$

Proof. Firstly, the boundedness of the sequences $\left\{x_{k}\right\}$ and $\left\{z_{k}\right\}$ has to be shown. Suppose $x^{*} \in \Omega$ is a solution of (1.1). Then by monotonicity of $g$, we have

$$
\begin{aligned}
\left(x_{k}-x^{*}\right)^{T} g\left(z_{k}\right) & =\left(x_{k}-z_{k}+z_{k}-x^{*}\right)^{T} g\left(z_{k}\right) \\
& =\left(x_{k}-z_{k}\right)^{T} g\left(z_{k}\right)+\left(z_{k}-x^{*}\right)^{T} g\left(z_{k}\right) \\
& \geq\left(x_{k}-z_{k}\right)^{T} g\left(z_{k}\right)+\left(z_{k}-x^{*}\right)^{T} g\left(x^{*}\right) \\
& =\left(x_{k}-z_{k}\right)^{T} g\left(z_{k}\right) .
\end{aligned}
$$

Also from (2.9), (2.86), and the fact that $0<\Lambda<2$, we have

$$
\begin{aligned}
\left\|x_{k+1}-x^{*}\right\|^{2} & =\left\|\Pi_{\Omega}\left(x_{k}-\Lambda \bar{\mu}_{k} g\left(z_{k}\right)\right)-x^{*}\right\|^{2} \\
& \leq\left\|x_{k}-\Lambda \bar{\mu}_{k} g\left(z_{k}\right)-x^{*}\right\|^{2} \\
& =\left\|\left(x_{k}-x^{*}\right)-\Lambda \bar{\mu}_{k} g\left(z_{k}\right)\right\|^{2} \\
& =\left\|x_{k}-x^{*}\right\|^{2}-2 \Lambda \bar{\mu}_{k} g\left(z_{k}\right)^{T}\left(x_{k}-x^{*}\right)+\Lambda^{2} \bar{\mu}_{k}^{2}\left\|g\left(z_{k}\right)\right\|^{2} \\
& \leq\left\|x_{k}-x^{*}\right\|^{2}-2 \Lambda \bar{\mu}_{k} g\left(z_{k}\right)^{T}\left(x_{k}-z_{k}\right)+\Lambda^{2} \bar{\mu}_{k}^{2}\left\|g\left(z_{k}\right)\right\|^{2} \\
& =\left\|x_{k}-x^{*}\right\|^{2}-\Lambda(2-\Lambda) \frac{\left(g\left(z_{k}\right)^{T}\left(x_{k}-z_{k}\right)\right)^{2}}{\left\|g\left(z_{k}\right)\right\|^{2}} \\
& \leq\left\|x_{k}-x^{*}\right\|^{2}
\end{aligned}
$$

which consequently reduces to

$$
\left\|x_{k+1}-x^{*}\right\| \leq\left\|x_{k}-x^{*}\right\|, \quad \forall k \geq 0 .
$$


And recursively, inequality (3.4) implies that $\left\|x_{k}-x^{*}\right\| \leq\left\|x_{0}-x^{*}\right\|, \forall k$. Hence, the sequence $\left\{\left\|x_{k}-x^{*}\right\|\right\}$ is decreasing and bounded, which also implies that $\left\{x_{k}\right\}$ is bounded. Also, using Assumption 2.2, (2.10) and (3.4) we obtain

$$
\left\|g\left(x_{k}\right)\right\|=\left\|g\left(x_{k}\right)-g\left(x^{*}\right)\right\| \leq L\left\|x_{k}-x^{*}\right\| \leq L\left\|x_{0}-x^{*}\right\|,
$$

where $L\left\|x_{0}-x^{*}\right\|=\pi$. Hence, we obtain that

$$
\left\|g\left(x_{k}\right)\right\| \leq \pi
$$

Also, using (2.7) and definition of $z_{k}$, we see that

$$
g\left(z_{k}\right)^{T}\left(x_{k}-z_{k}\right)=-t_{k} g\left(z_{k}\right)^{T} d_{k} \geq \eta t_{k}^{2}\left\|d_{k}\right\|^{2}=\eta\left\|x_{k}-z_{k}\right\|^{2} .
$$

Utilizing the monotonicity of $g$ and the Cauchy-Schwartz inequality, we can write

$$
\begin{aligned}
g\left(z_{k}\right)^{T}\left(x_{k}-z_{k}\right) & =\left(g\left(z_{k}\right)-g\left(x_{k}\right)\right)^{T}\left(x_{k}-z_{k}\right)+g\left(x_{k}\right)^{T}\left(x_{k}-z_{k}\right) \\
& \leq\left\|g\left(x_{k}\right)\right\|\left\|x_{k}-z_{k}\right\| .
\end{aligned}
$$

By (3.6), (3.7) and (3.8) we get

$$
\eta\left\|x_{k}-z_{k}\right\|^{2} \leq\left\|g\left(x_{k}\right)\right\|\left\|x_{k}-z_{k}\right\|
$$

which leads to

$$
\left\|x_{k}-z_{k}\right\| \leq \frac{\pi}{\eta}
$$

Therefore, the sequence $\left\{z_{k}\right\}$ is also bounded.

Moreover, the boundedness of $\left\{z_{k}\right\}$, implies that $\left\{\left\|z_{k}-x^{*}\right\|\right\}$ is bounded, namely there exists $\tau>0$ such that for any $x^{*} \in \Omega$

$$
\left\|z_{k}-x^{*}\right\| \leq \tau .
$$

Also, from (2.10) and (3.11), we have

$$
\left\|g\left(z_{k}\right)\right\|=\left\|g\left(z_{k}\right)-g\left(x^{*}\right)\right\| \leq L\left\|z_{k}-x^{*}\right\| \leq L \tau=\Psi .
$$

Hence

$$
\left\|g\left(z_{k}\right)\right\| \leq \Psi
$$

Also, using the line search condition (2.7), we have

$$
\eta^{2} t_{k}^{4}\left\|d_{k}\right\|^{4} \leq t_{k}^{2}\left(g\left(z_{k}\right)^{T} d_{k}\right)^{2}
$$

By combining (3.3) and (3.14), we get

$$
\eta^{2} t_{k}^{4}\left\|d_{k}\right\|^{4} \leq \frac{\left\|g\left(z_{k}\right)\right\|^{2}}{\Lambda(2-\Lambda)}\left(\left\|x_{k}-x^{*}\right\|^{2}-\left\|x_{k+1}-x^{*}\right\|^{2}\right) .
$$

Now, from (3.3) we obtain that the sequence $\left\{\left\|x_{k}-x^{*}\right\|\right\}$ is convergent, and $\left\{g\left(z_{k}\right)\right\}$ is also bounded by (3.13). So, taking limits of both sides of (3.15) as $k$ grows to infinity, we obtain

$$
\eta^{2} \lim _{k \rightarrow \infty} t_{k}^{4}\left\|d_{k}\right\|^{4} \leq 0,
$$

for which we get

$$
\lim _{k \rightarrow \infty} t_{k}\left\|d_{k}\right\|=0 .
$$


Lemma 3.2. . Let Assumption 2.3 hold. Then there exists a constant $\tilde{\varpi}>0$ such that

$$
\left\|d_{k}\right\| \leq \tilde{\varpi}, \quad \forall k .
$$

Proof. Considering (2.53) two cases are analyzed:

Case 1. $\bar{\theta}_{k 1}>\vartheta$. Then $\theta_{k 1}^{*}=\bar{\theta}_{k 1}$. So, using (2.52) and the Cauchy-Schwartz inequality, we get

$$
\left|\bar{\theta}_{k 1}\right|=2\left|\frac{\left(s_{k-1}^{T} \tilde{y}_{k-1}\right)^{2}}{\left\|s_{k-1}\right\|^{2}\left\|\tilde{y}_{k-1}\right\|^{2}}\right| \leq 2 \frac{\left\|s_{k-1}\right\|^{2}\left\|\tilde{y}_{k-1}\right\|^{2}}{\left\|s_{k-1}\right\|^{2}\left\|\tilde{y}_{k-1}\right\|^{2}}=2 .
$$

Also using (2.10), (2.28), (2.54), (2.55), (3.6), (3.19) and the Cauchy-Schwartz inequality, we obtain

$$
\begin{aligned}
\left\|d_{k}\right\| & =\left\|-g_{k}+\beta_{k}^{\theta_{k 1}^{*}} s_{k-1}\right\| \\
& =\left\|-g_{k}+\frac{g_{k}^{T} \tilde{y}_{k-1}}{s_{k-1}^{T} \tilde{y}_{k-1}} s_{k-1}-\bar{\theta}_{k 1} \frac{\left\|\tilde{y}_{k-1}\right\|^{2} g_{k}^{T} s_{k-1}}{\left(s_{k-1}^{T} \tilde{y}_{k-1}\right)^{2}} s_{k-1}\right\| \\
& \leq\left\|g_{k}\right\|+\frac{\left\|g_{k}\right\|\left\|\tilde{y}_{k-1}\right\|}{s_{k-1}^{T} \tilde{y}_{k-1}}\left\|s_{k-1}\right\|+\left|\bar{\theta}_{k 1}\right| \frac{\left\|\tilde{y}_{k-1}\right\|^{2}\left\|g_{k}\right\|\left\|s_{k-1}\right\|}{\left(s_{k-1}^{T} \tilde{y}_{k-1}\right)^{2}}\left\|s_{k-1}\right\| \\
& \leq \pi+\frac{L \pi\left\|s_{k-1}\right\|^{2}}{c\left\|s_{k-1}\right\|^{2}}+2 \frac{L^{2} \pi\left\|s_{k-1}\right\|^{4}}{c^{2}\left\|s_{k-1}\right\|^{4}} \\
& =\pi+\frac{L \pi}{c}+2 \frac{L^{2} \pi}{c^{2}} \\
& =\left(1+\frac{L}{c}+2 \frac{L^{2}}{c^{2}}\right) \pi=\varpi_{1} .
\end{aligned}
$$

Case 2. $\bar{\theta}_{k 1}<\vartheta$. Then $\theta_{k 1}^{*}=\vartheta$. So, using (2.10), (2.28), (2.54), (2.55), (3.6), and the Cauchy-Schwartz inequality, we obtain

$$
\begin{aligned}
\left\|d_{k}\right\| & =\left\|-g_{k}+\beta_{k}^{\theta_{k 1}^{*}} s_{k-1}\right\| \\
& =\left\|-g_{k}+\frac{g_{k}^{T} \tilde{y}_{k-1}}{s_{k-1}^{T} \tilde{y}_{k-1}} s_{k-1}-(\vartheta) \frac{\left\|\tilde{y}_{k-1}\right\|^{2} g_{k}^{T} s_{k-1}}{\left(s_{k-1}^{T} \tilde{y}_{k-1}\right)^{2}} s_{k-1}\right\| \\
& \leq\left\|g_{k}\right\|+\frac{\left\|g_{k}\right\|\left\|\tilde{y}_{k-1}\right\|}{s_{k-1}^{T} \tilde{y}_{k-1}}\left\|s_{k-1}\right\|+|\vartheta| \frac{\left\|\tilde{y}_{k-1}\right\|^{2}\left\|g_{k}\right\|\left\|s_{k-1}\right\|}{\left(s_{k-1}^{T} \tilde{y}_{k-1}\right)^{2}}\left\|s_{k-1}\right\| \\
& \leq \pi+\frac{L \pi\left\|s_{k-1}\right\|}{c\left\|s_{k-1}\right\|^{2}}\left\|s_{k-1}\right\|+\vartheta \frac{L^{2} \pi\left\|s_{k-1}\right\|^{2}\left\|s_{k-1}\right\|}{c^{2}\left\|s_{k-1}\right\|^{4}}\left\|s_{k-1}\right\| \\
& =\pi+\frac{L \pi}{c}+\vartheta \frac{L^{2} \pi}{c^{2}} \\
& =\left(1+\frac{L}{c}+\vartheta \frac{L^{2}}{c^{2}}\right) \pi=\varpi_{2} .
\end{aligned}
$$

Therefore, by setting $\tilde{\varpi}=\max \left\{\varpi_{1}, \varpi_{2}\right\}$ we establish the result.

The subsequent theorem is used to prove global convergence of Algorithm 1. 
Theorem 3.3. Let Assumptions 2.1-2.3 hold. Consider the sequences $\left\{x_{k}\right\}$ and $\left\{z_{k}\right\}$ generated by Algorithm 1 . Then

$$
\liminf _{k \rightarrow \infty}\left\|g\left(x_{k}\right)\right\|=0 .
$$

Proof. The proof takes the form of contradiction. Suppose (3.22) does not hold, then it implies there exists $\varepsilon_{0}>0$ such that

$$
\left\|g\left(x_{k}\right)\right\| \geq \varepsilon_{0} \quad \text { holds, } \quad \forall k .
$$

Two cases are analyzed here:

(1) If $\liminf \operatorname{in}_{k \rightarrow \infty} t_{k}>0$, then by (2.56) and (3.17), we get $\liminf _{k \rightarrow \infty}\left\|g\left(x_{k}\right)\right\|=0$, which is a contradiction with (3.23).

(2) On the other hand, if $\liminf _{k \rightarrow \infty} t_{k}=0$, namely an infinite indexing set $\mathbf{K}$ exists such that

$$
\lim _{k \in \mathbf{K}, k \rightarrow \infty} t_{k}=0 .
$$

Then from (2.7), and for $k \in \mathbf{K}$ sufficiently large, if $t_{k} \neq \gamma$, then $\gamma^{-1} t_{k}$ does not satisfy (2.7), namely

$$
-g\left(x_{k}+\gamma^{-1} t_{k} d_{k}\right)^{T} d_{k}<\eta \gamma^{-1} t_{k}\left\|d_{k}\right\|^{2} .
$$

Also, using Assumption 2.3, (2.56), and (2.7), we can write

$$
\begin{aligned}
\Phi\left\|g\left(x_{k}\right)\right\|^{2} & \leq-g\left(x_{k}\right)^{T} d_{k} \\
& =g\left(x_{k}+\gamma^{-1} t_{k} d_{k}-g_{k}\right)^{T} d_{k}-g_{k}^{T} d_{k}-g\left(x_{k}+\gamma^{-1} t_{k} d_{k}\right)^{T} d_{k} \\
& \leq \gamma^{-1} t_{k}(L+\eta)\left\|d_{k}\right\|^{2} .
\end{aligned}
$$

So,

$$
t_{k}\left\|d_{k}\right\| \geq \frac{\Phi \gamma\left\|g_{k}\right\|^{2}}{(L+\eta)\left\|d_{k}\right\|}
$$

From both sides of the last inequality, we take limits as $k$ grows to infinity to obtain a contradiction with (3.17), which implies that $\liminf _{k \rightarrow \infty}\left\|g\left(x_{k}\right)\right\|=0$.

Since Lemma 3.1 also holds for Algorithm 2, we only need to show boundedness of the search direction generated by Algorithm 2 .

Lemma 3.4. Suppose Assumption 2.3 holds. Let the bounded sequence $\left\{x_{k}\right\}$ be generated by Algorithm 1. Then there exists a constant $\hat{M}>0$ such that

$$
\left\|d_{k}\right\| \leq \hat{M}, \quad \forall k
$$

Proof. Here, we also consider the two possible cases outlined from (2.82):

Case 1. $\bar{\theta}_{k 2}>\sigma$. Then $\theta_{k 2}^{*}=\bar{\theta}_{k 2}$. So, using (2.81) and the Cauchy-Schwarz inequality, we get

$$
\left|\bar{\theta}_{k 2}\right|=\left|\frac{s_{k-1}^{T} \tilde{y}_{k-1}}{\left\|s_{k-1}\right\|\left\|\tilde{y}_{k-1}\right\|}\right| \leq \frac{\left\|s_{k-1}\right\|\left\|\tilde{y}_{k-1}\right\|}{\left\|s_{k-1}\right\|\left\|\tilde{y}_{k-1}\right\|}=1 .
$$


Also using (2.10), (2.28), (2.83), (2.84), (3.6), (3.29), and the Cauchy-Schwartz inequality, we obtain

$$
\begin{aligned}
\left\|d_{k}\right\| & =\left\|-g_{k}+\beta_{k}^{\theta_{2}^{*}} s_{k-1}\right\| \\
& =\left\|-g_{k}+\frac{g_{k}^{T} \tilde{y}_{k-1}}{s_{k-1}^{T} \tilde{y}_{k-1}} s_{k-1}-\bar{\theta}_{k 2} \frac{\left\|\tilde{y}_{k-1}\right\|^{2} g_{k}^{T} s_{k-1}}{\left(s_{k-1}^{T} \tilde{y}_{k-1}\right)^{2}} s_{k-1}\right\| \\
& \leq\left\|g_{k}\right\|+\frac{\left\|g_{k}\right\|\left\|\tilde{y}_{k-1}\right\|}{s_{k-1}^{T} \tilde{y}_{k-1}}\left\|s_{k-1}\right\|+\left|\bar{\theta}_{k 2}\right| \frac{\left\|\tilde{y}_{k-1}\right\|^{2}\left\|g_{k}\right\|\left\|s_{k-1}\right\|}{\left(s_{k-1}^{T} \tilde{y}_{k-1}\right)^{2}}\left\|s_{k-1}\right\| \\
& \leq \pi+\frac{L \pi\left\|s_{k-1}\right\|^{2}}{c\left\|s_{k-1}\right\|^{2}}+\frac{L^{2} \pi\left\|s_{k-1}\right\|^{2}\left\|s_{k-1}\right\|^{2}}{c^{2}\left\|s_{k-1}\right\|^{4}} \\
& =\pi+\frac{L \pi}{c}+\frac{L^{2} \pi}{c^{2}} \\
& =\left(1+\frac{L}{c}+\frac{L^{2}}{c^{2}}\right) \pi=\hat{M}_{1} .
\end{aligned}
$$

Case 2. $\bar{\theta}_{k 2}<\sigma$. Then by (2.82), $\theta_{2}^{*}=\sigma$. So, using (2.10), (2.28), (2.83), (2.84), (3.6), and the Cauchy-Schwartz inequality, we obtain

$$
\begin{aligned}
\left\|d_{k}\right\| & =\left\|-g_{k}+\beta_{k}^{\theta_{2}^{*}} s_{k-1}\right\| \\
& =\left\|-g_{k}+\frac{g_{k}^{T} \tilde{y}_{k-1}}{s_{k-1}^{T} \tilde{y}_{k-1}} s_{k-1}-\sigma \frac{\left\|\tilde{y}_{k-1}\right\|^{2} g_{k}^{T} s_{k-1}}{\left(s_{k-1}^{T} \tilde{y}_{k-1}\right)^{2}} s_{k-1}\right\| \\
& \leq\left\|g_{k}\right\|+\frac{\left\|g_{k}\right\|\left\|\tilde{y}_{k-1}\right\|}{s_{k-1}^{T} \tilde{y}_{k-1}}\left\|s_{k-1}\right\|+|\sigma| \frac{\left\|\tilde{y}_{k-1}\right\|^{2}\left\|g_{k}\right\|\left\|s_{k-1}\right\|}{\left(s_{k-1}^{T} \tilde{y}_{k-1}\right)^{2}}\left\|s_{k-1}\right\| \\
& \leq \pi+\frac{L \pi\left\|s_{k-1}\right\|^{2}}{c\left\|s_{k-1}\right\|^{2}}+\sigma \frac{L^{2} \pi\left\|s_{k-1}\right\|^{4}}{c^{2}\left\|s_{k-1}\right\|^{4}} \\
& =\pi+\frac{L \pi}{c}+\sigma \frac{L^{2} \pi}{c^{2}} \\
& =\left(1+\frac{L}{c}+\sigma \frac{L^{2}}{c^{2}}\right) \pi=\hat{M}_{2} .
\end{aligned}
$$

Therefore, by setting $\hat{M}=\max \left\{\hat{M}_{1}, \hat{M}_{2}\right\}$, the required result is obtained.

We now employed the following theorem for global convergence of Algorithm 2.

Theorem 3.5. Let the sequence $\left\{x_{k}\right\}$ be generated by Algorithm 2. Then

$$
\liminf _{k \rightarrow \infty}\left\|g\left(x_{k}\right)\right\|=0 .
$$

The proof follows the same pattern as that of Algorithm 1. Since the directions of Algorithm 2 also satisfy (2.56), using similar arguments as for Algorithm 1, the result is obtained.

\section{NUMERICAL EXPERIMENTS AND COMPARISONS}

At this juncture, the report of a set of numerical experiments is presented to exhibit performance and efficiency of the methods proposed. We test the performances of Algorithm 1 (NIHZPM) and Algorithm 2 (NEHZPM) 
with the modified HZ methods in [61,78,83] and the efficient projected gradient method in [39], which are called MHZM1, MHZM2, CGD and EPGM for simplicity. For all the six methods, the backtracking line search defined in (2.7) was used, and the parameters for Algorithms 1 and 2 are set as follows:

- Algorithm 1: $\eta=10^{-2}, \gamma=0.48, \bar{\sigma}=0.6, \vartheta=1.2, c=1, \Lambda=1.9$.

- Algorithm 2: $\eta=10^{-7}, \gamma=0.48, \bar{\sigma}=0.6, \sigma=0.28, c=0.001, \Lambda=1.9$.

For the MHZM1, MHZM2, CGD and EPGM methods, the parameters are set as they are used by the respective authors. In addition, the codes used for implementation of all the algorithms were done in Matlab R2015a platform, and run on a personal computer with configuration (CPU 1.8 GHz, 4GB memory). The iteration process terminates when it exceeds 1000 or $\left\|g\left(x_{k}\right)\right\| \leq \epsilon$ or $\left\|g\left(z_{k}\right)\right\| \leq \epsilon$, with $\epsilon=10^{-8}$.

Also, reports of the experiments carried out are presented in Tables 1-6, where "Pnum" and "NVars" stands for problem solved and number of variables for each problem, while "Ipt" and "Inum" denotes initial guess and the total iterations achieved respectively. "Fval" and "Ptime" represent number of functions values and processing time respectively. The residual at stopping point is represented by "Norm", while "**" indicate failure of a method to converge to a solution.

The following problems were used, where $g(x)=\left(g_{1}(x), g_{2}(x), \ldots, g_{n}(x)\right)^{T}$.

Problem 4.1. The Logarithmic Function obtained from [83].

$g_{i}(x)=\log \left(x_{i}+1\right)-\frac{x_{i}}{n}, \quad i=2, \ldots n$,

where $\Omega=\mathbf{R}_{+}^{n}$.

Problem 4.2. Exponential Function obtained from [43].

$g_{1}(x)=e^{x_{i}}-1$,

$g_{i}(x)=\frac{i}{10}\left(e^{x_{i}}+x_{i-1}-1\right), \quad i=2,3, \ldots, n$,

where $\Omega=\mathbf{R}_{+}^{n}$.

Problem 4.3. Non-smooth Function [82].

$g_{i}(x)=x_{i}-\sin \left|x_{i}-1\right|, \quad i=1,2, \ldots, n$,

where $\Omega=\left\{x \in \mathbf{R}^{n}: \sum_{i=1}^{n} x_{i} \leq n, \quad x_{i} \geq-1, \quad i=1,2, \ldots, n\right\}$.

Problem 4.4. A modification of Problem 4.2 in [82].

$g_{i}(x)=x_{i}-2 \sin \left|x_{i}-1\right|, \quad i=1,2, \ldots, n$,

where $\Omega=\left\{x \in \mathbf{R}^{n}: \sum_{i=1}^{n} x_{i} \leq n, \quad x_{i} \geq-1, \quad i=1,2, \ldots, n\right\}$.

Problem 4.5. Tridiagonal Exponential Function [46].

$g_{1}(x)=x_{1}-e^{\left(\cos \frac{x_{1}+x_{2}}{n+1}\right)}$,

$g_{i}(x)=x_{i}-e^{\left(\cos \frac{x_{i-1}+x_{i}+x_{i+1}}{n+1}\right)}, \quad i=2,3, \ldots, n-1$,

$g_{n}(x)=x_{n}-e^{\left(\cos \frac{x_{n-1}+x_{n}}{n+1}\right)}$.

where $\Omega=\mathbf{R}_{+}^{n}$.

Problem 4.6. Exponential Function [43].

$g_{1}(x)=e^{x_{1}}-1$,

$g_{i}(x)=e^{x_{i}}+x_{i}-1, \quad i=2, \ldots, n$,

where $\Omega=\mathbf{R}_{+}^{n}$.

Problem 4.7. [54].

$g_{1}(x)=-2 x_{1}-x_{2}+e^{x_{1}}-1$,

$g_{i}(x)=2 x_{i}-x_{i-1}-x_{i+1}+e^{x_{i}}-1, \quad i=2,3, \ldots, n-1$,

$g_{n}(x)=2 x_{n}-x_{n-1}+e^{x_{n}}-1$.

where $\Omega=\left\{x \in \mathbf{R}^{n}: \sum_{i=1}^{n} x_{i} \leq n, \quad x_{i} \geq 0, \quad i=1, \ldots, n\right\}$. 


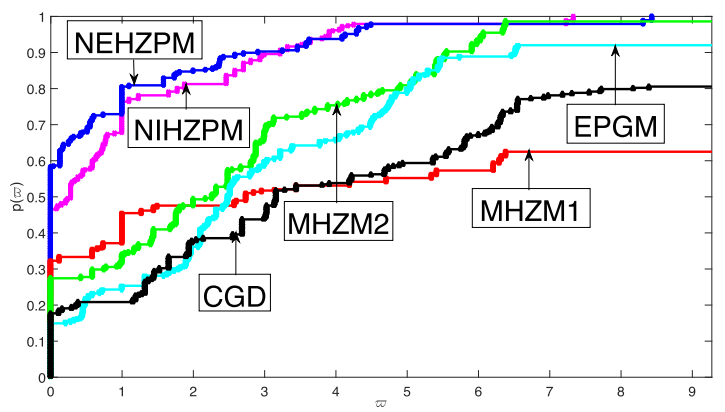

FIGURE 1. Iterations performance profiles for the six methods.

Problem 4.8. Modification of Problem 4.5 in [18].

$g_{i}(x)=2 \phi\left(x_{i}-1\right)+4\left(\sum_{j=1}^{n} x_{j}-0.25\right) x_{i}, \quad i=1,2, \ldots, n$,

where $\phi=10^{-5}, \quad \Omega=\mathbf{R}_{+}^{n}$.

Problem 4.9. Non-smooth Function [42].

$g_{i}(x)=2 x_{i}-\sin \left|x_{i}\right|, \quad i=1,2, \ldots, n$,

where $\Omega=\mathbf{R}_{+}^{n}$.

Problem 4.10. [66].

$g_{i}(x)=e^{x_{i}}-2, \quad i=2, \ldots, n$,

where $\Omega=\mathbf{R}_{+}^{n}$.

Problem 4.11. [42].

$g_{i}(x)=\min \left\{\min \left\{\left|x_{i}\right|, x_{i}^{2}\right\}, \max \left\{\left|x_{i}\right|, x_{i}^{3}\right\}\right\}, \quad i=1,2, \ldots n$,

where $\Omega=\mathbf{R}_{+}^{n}$.

Problem 4.12. Troesch problem obtained from [43].

$g_{1}(x)=2 x_{1}+\varrho \hbar^{2} \sinh \left(\varrho x_{1}\right)-x_{2}$

$g_{i}(x)=2 x_{i}+\varrho \hbar^{2} \sinh \left(\varrho x_{i}\right)-x_{i-1}-x_{i+1}, \quad i=2,3, \ldots, n-1$,

$g_{n}(x)=2 x_{n}+\varrho \hbar^{2} \sinh \left(\varrho x_{n}\right)-x_{n-1}$.

where $\hbar=\frac{1}{n+1}, \varrho=10, \Omega=\mathbf{R}_{+}^{n}$.

Clearly, Problems 4.3, 4.4 and 4.9 are nonsmooth at $x=(1,1, \ldots, 1)^{T}, x=(1,1, \ldots, 1)^{T}$, and $x=(0,0, \ldots, 0)^{T}$ respectively. For each of the above test functions, 24 numerical experiments were performed with variables 1000 , 5000 , and 10000 , and the following starting points:

$$
\begin{aligned}
& x 1=\left(1-\frac{1}{n}, 1-\frac{2}{n}, \ldots, 0\right)^{T}, \quad x 2=\left(4,2, \ldots, \frac{-2\left(-1^{n}-3\right)}{2}\right)^{T}, \quad x 3=\left(n-\frac{1}{n}, n-\frac{2}{n}, \ldots, n-1\right)^{T}, \\
& x 4=\left(1, \frac{1}{2}, \ldots, \frac{1}{n}\right)^{T}, \quad x 5=(4,4, \ldots, 4)^{T}, \quad x 6=(5,5, \ldots, 5)^{T}, \quad x 7=(7,7, \ldots, 7)^{T}, \quad x 8=(8,8, \ldots, 8)^{T} .
\end{aligned}
$$

Careful study of results displayed in Tables 1-6 reveals that the NIHZPM and NEHZPM methods solve all problems successfully, and more problems with minimum number of iterations and processing time than the MHZM1, MHZM2, EPGM and CGD methods, which also failed to solve some of the problems considered. In addition, a summary of the reported results from Tables 1 to 6 is presented in Table 7 to outline performance of each of the six methods in relation to number of iterations, function evaluations, and processing time. It is 


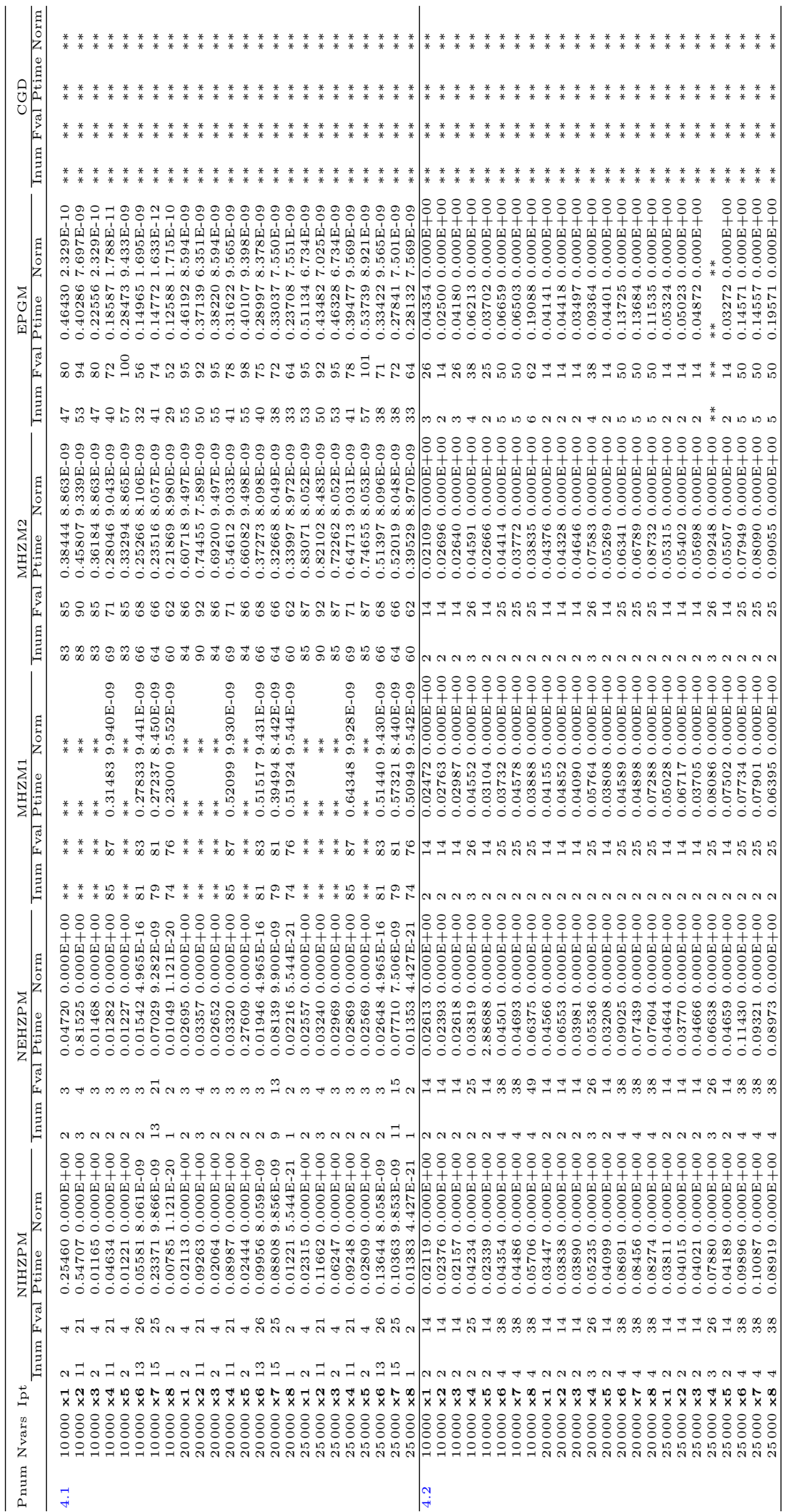




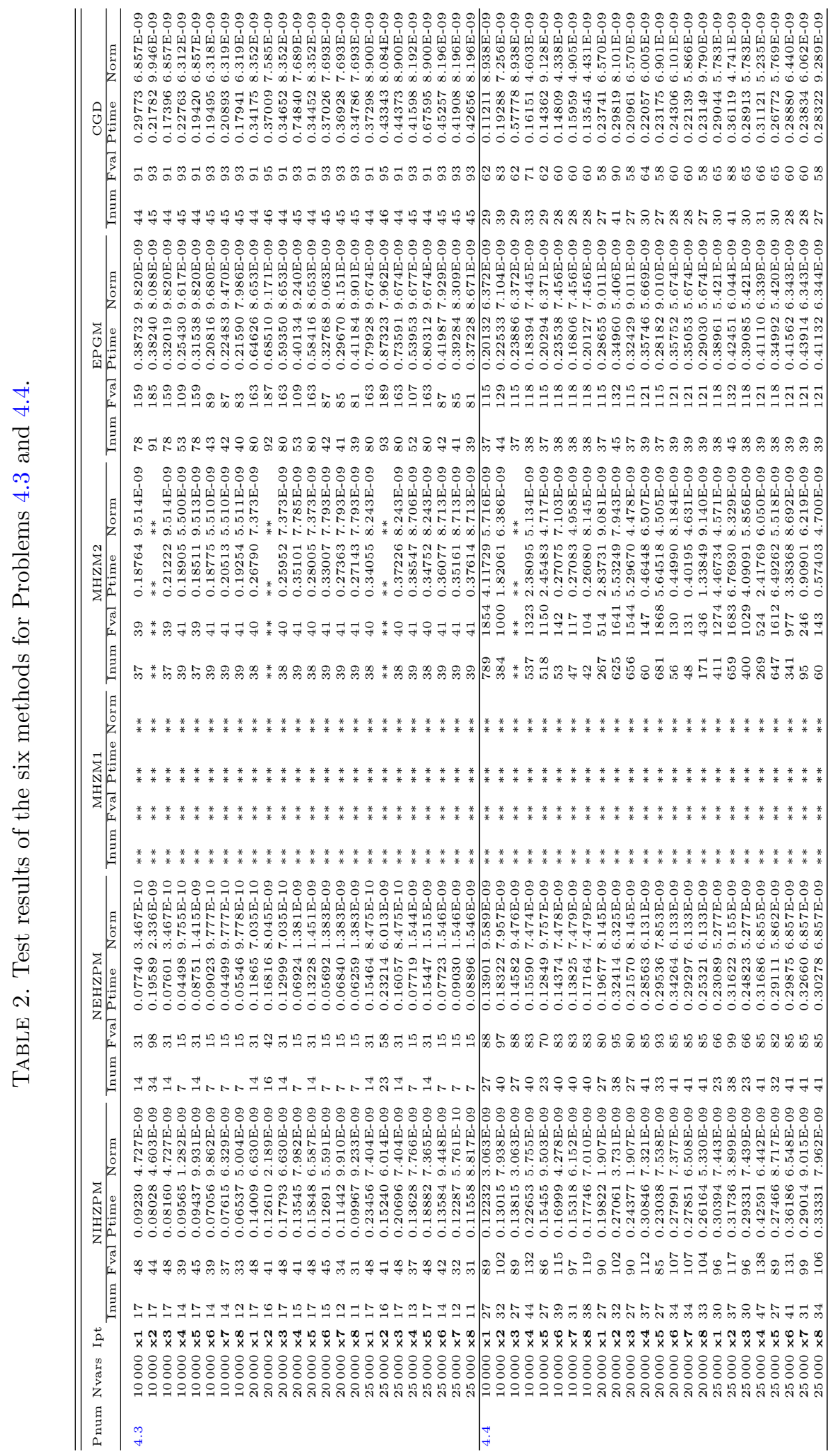




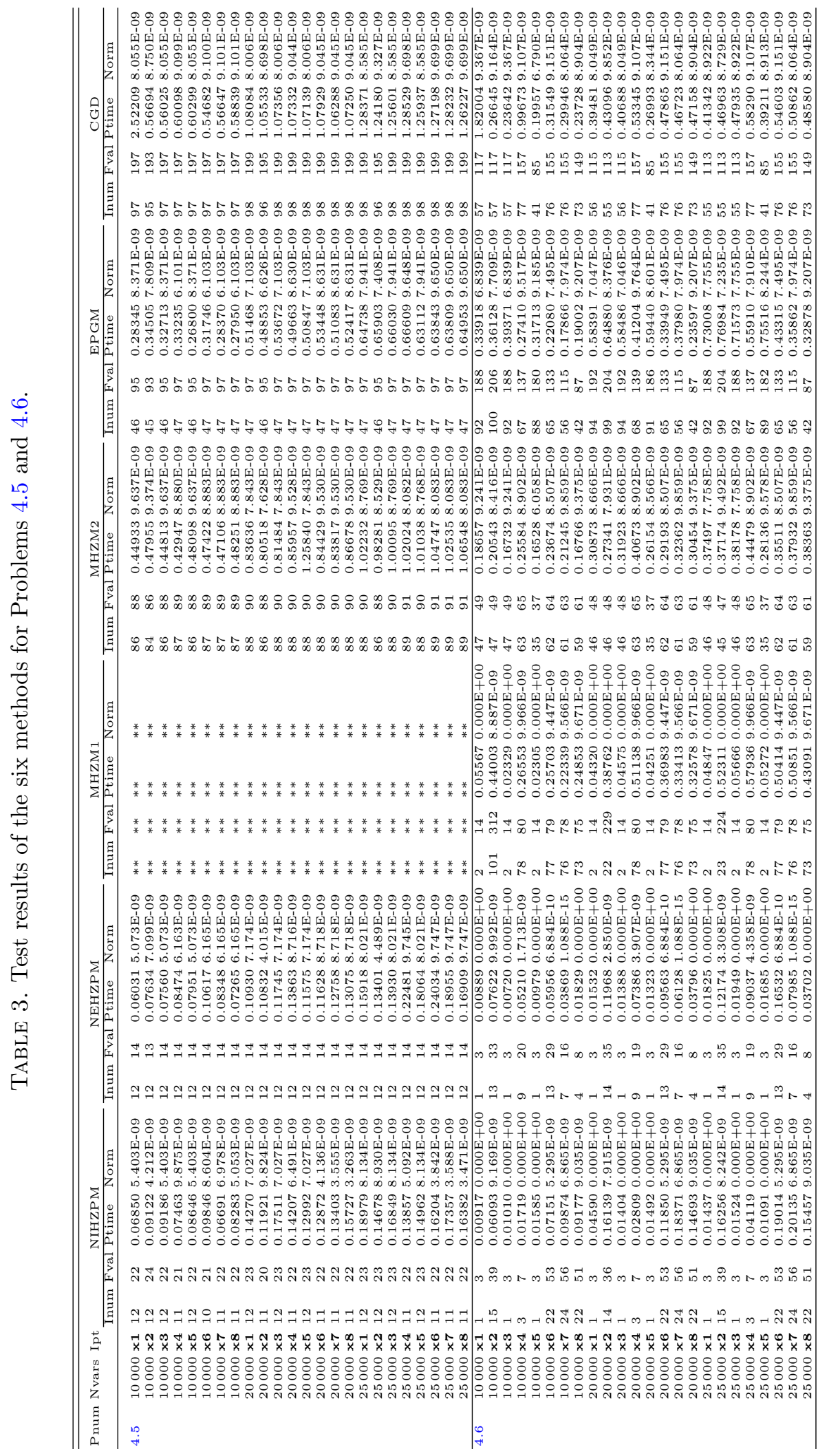




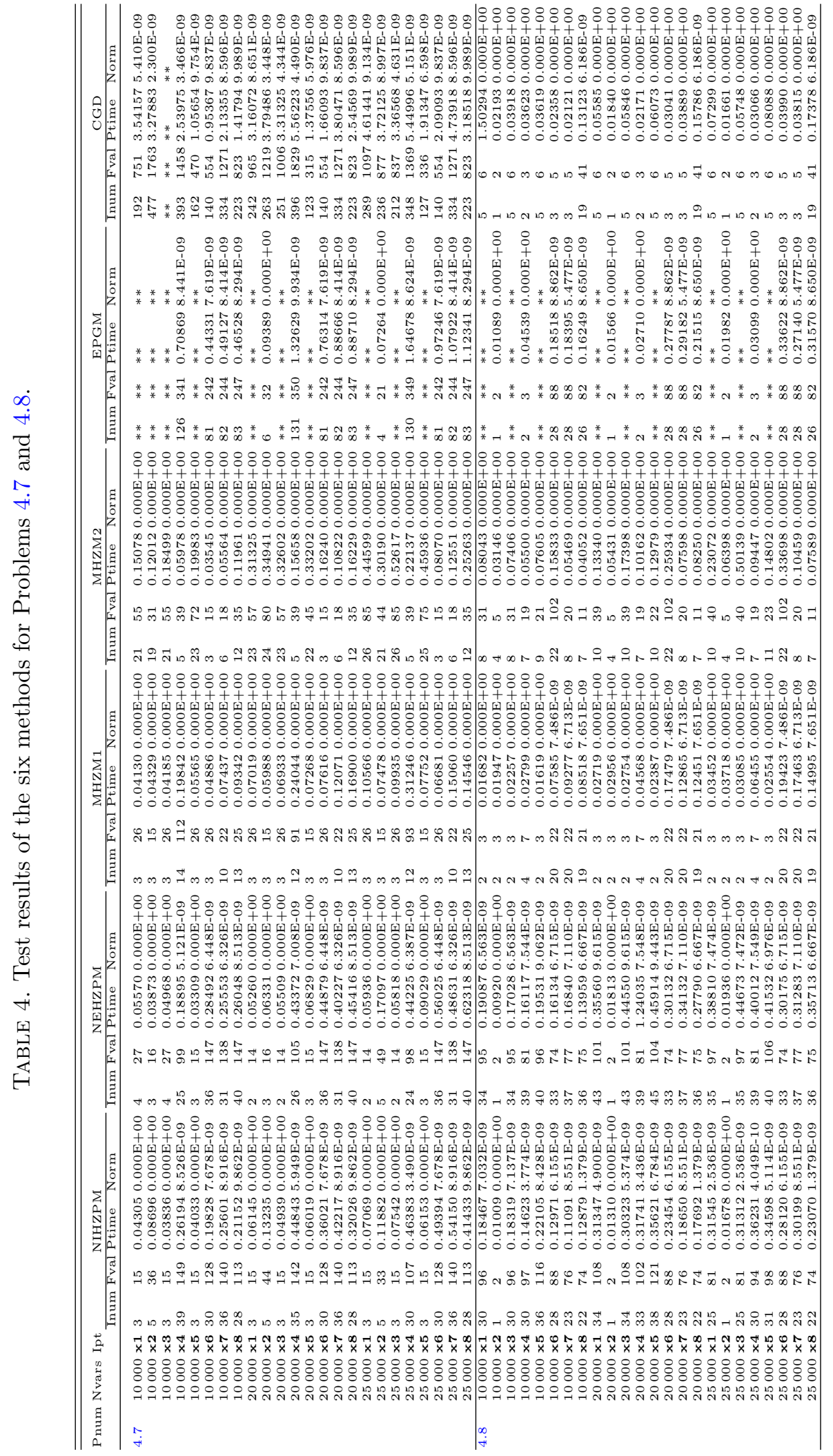




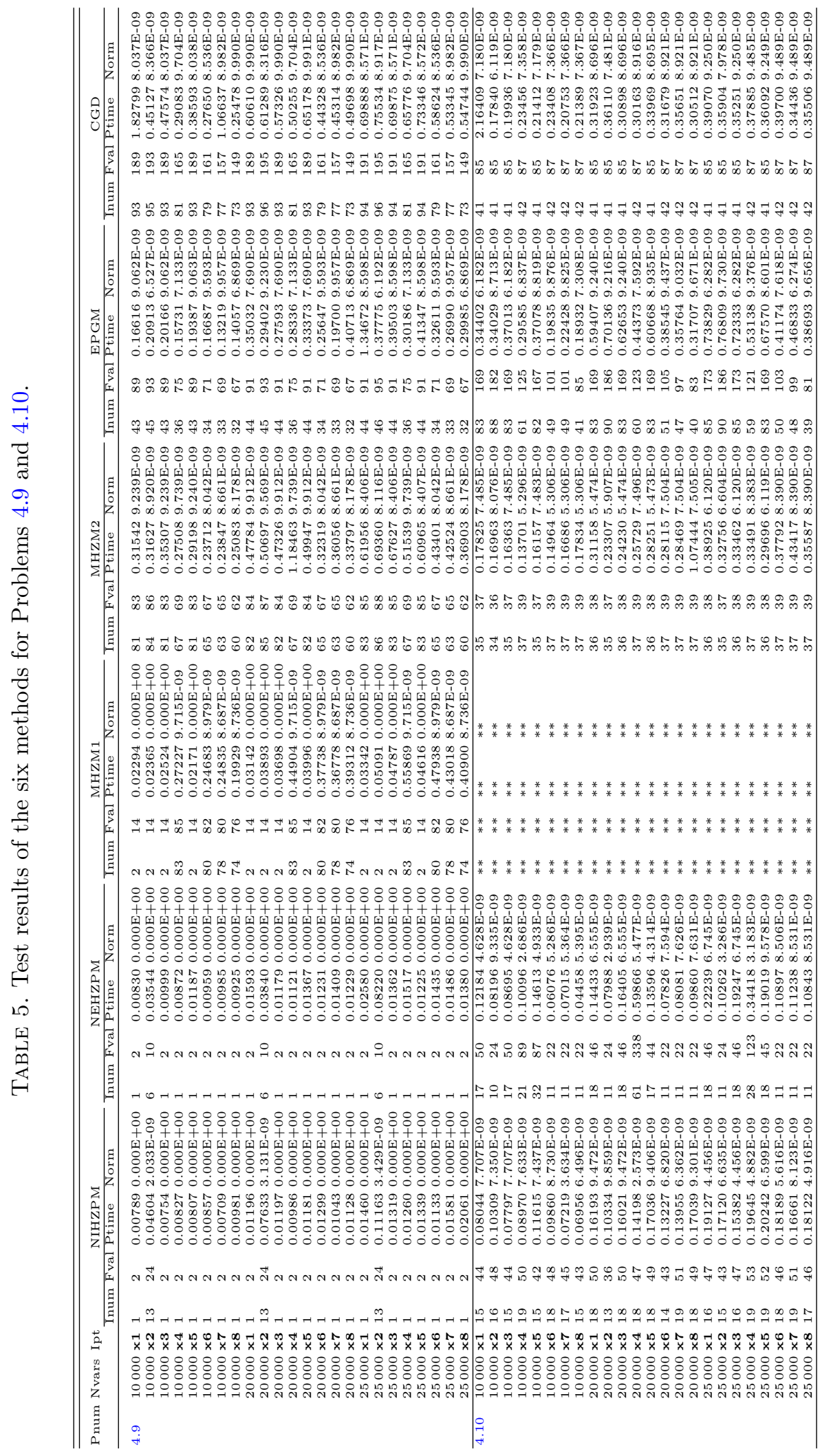




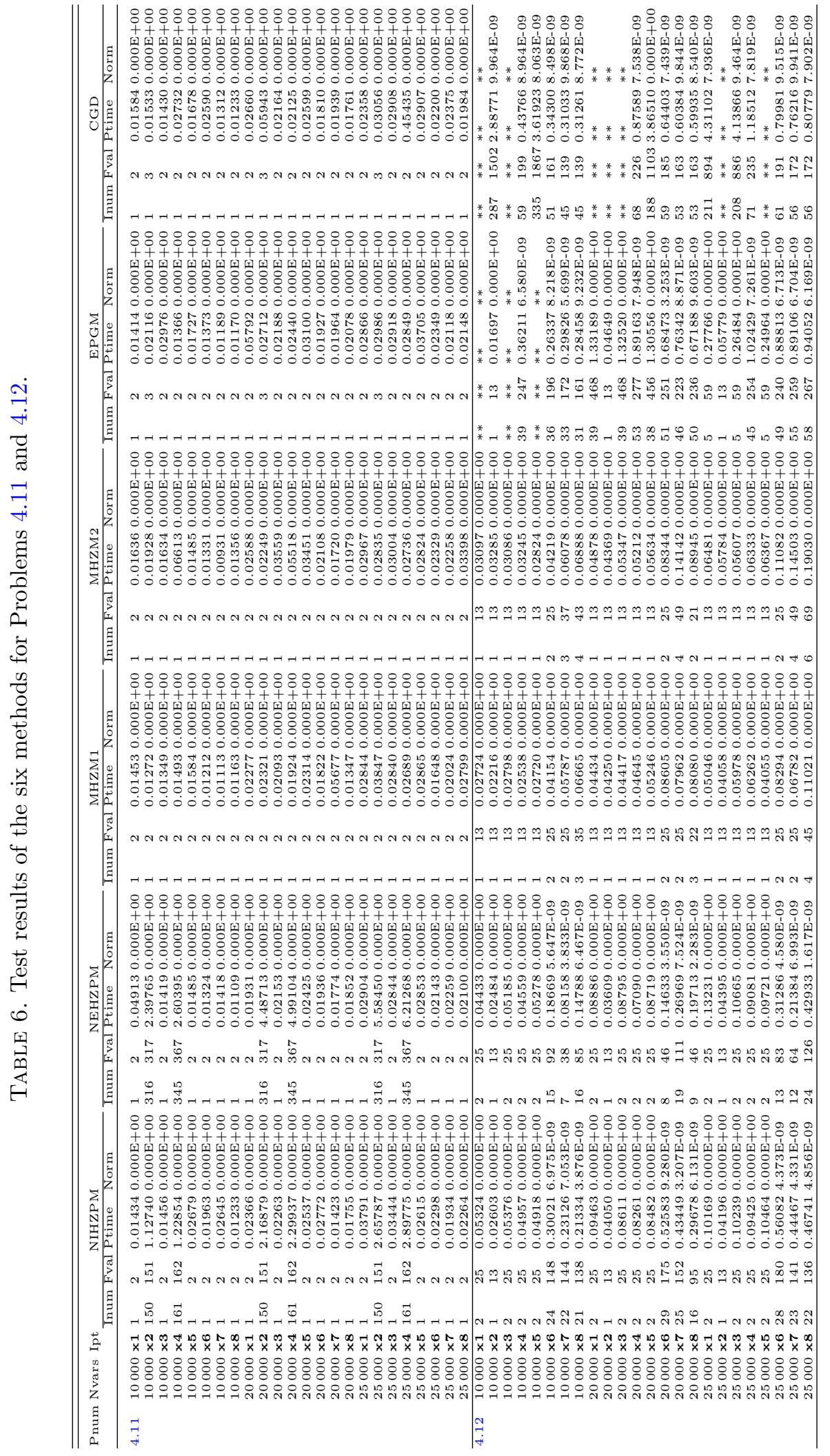


TABLE 7. Summary of results from Tables 1 to 6 displaying least number of iterations, function values and processing time by the six methods.

\begin{tabular}{lllllll}
\hline \hline Method & Inum & Percentage & Fval & Percentage & Ptime & Percentage \\
\hline NIHZPM & 30 & $10.42 \%$ & 8 & $2.78 \%$ & 70 & $24.31 \%$ \\
NEHZPM & 65 & $22.57 \%$ & 97 & $33.68 \%$ & 111 & $38.54 \%$ \\
MHZM1 & 19 & $6.60 \%$ & 19 & $6.60 \%$ & 56 & $19.45 \%$ \\
MHZM2 & 13 & $4.51 \%$ & 25 & $8.68 \%$ & 20 & $6.94 \%$ \\
EPGM & 0 & $0 \%$ & 0 & $0 \%$ & 7 & $2.43 \%$ \\
CGD & 18 & $6.25 \%$ & 30 & $10.42 \%$ & 24 & $8.33 \%$ \\
Undecided & 143 & $49.65 \%$ & 109 & $37.84 \%$ & 0 & $0 \%$ \\
\hline
\end{tabular}

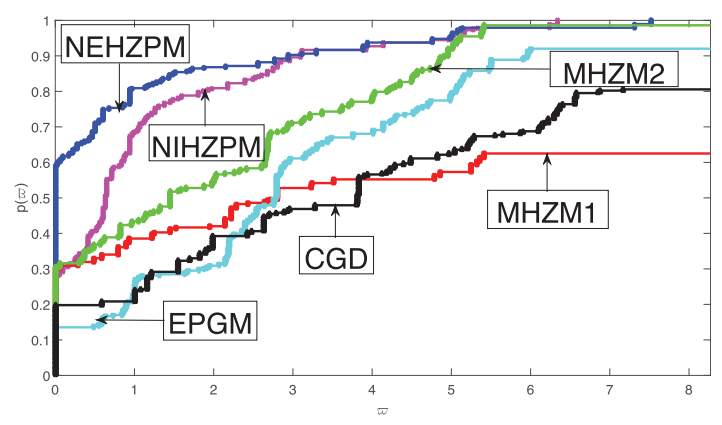

FIGURE 2. Function values performance profiles for the six methods.

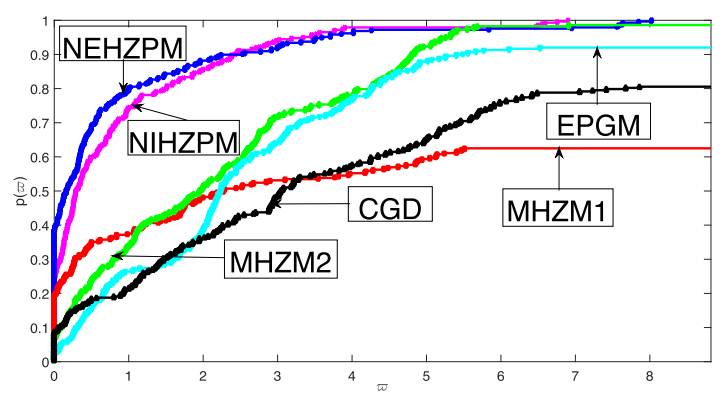

FiguRE 3. Processing time performance profiles for the six methods.

observed from the summary table that the NIHZPM and NEHZPM methods solved $10.42 \%$ (30 out of 288) and $22.57 \%$ (65 out of 288) of all the problems in the conducted experiments with least number of iterations compared to the MHZM1, MHZM2, EPGM and CGD methods, which recorded $6.60 \%$ (19 out of 288), $4.51 \%$ (13 out of 288 ), $0 \%$ ( 0 out of 288 ), and $6.25 \%$ (18 out of 288 ) respectively. The table also shows that $49.65 \%$ (143 out of 288) of the problems were solved by either $2,3,4,5$ or all six methods involved in the experiments with the same number of iterations. The summary table also shows that the NIHZPM and NEHZPM methods solved $2.78 \%$ ( 8 out of 288 ) and $33.68 \%$ (97 out of 288 ) of all the problems with minimum function evaluations compared to the MHZM1, MHZM2, EPGM and CGD methods, which recorded $6.60 \%$ (19 out of 288), 8.68\% ( 25 out of 288 ), $0 \%$ ( 0 out of 288 ), and $10.42 \%$ (30 out of 288 ) respectively. Moreover, the table indicates that $37.84 \%$ (109 out of 288 ) of the problems were solved with equal number of function evaluations by either $2,3,4,5$ or all six methods in the experiments. Finally, it is observed from the summarized results that the NIHZPM 
and NEHZPM methods solved 24.31\% (70 out of 288 ) and $38.54 \%$ (111 out of 288 ) of all the problems with least processing time as against the MHZM1, MHZM2, EPGM and CGD methods, which solved $19.45 \%$ (56 out of 288 ), $6.94 \%$ (20 out of 288 ), $2.43 \%$ (7 out of 288 ), and $8.33 \%$ (24 out of 288 ) respectively.

Remark 4.13. As an important observation, Tables 1-6 reveals the impact of the eight starting points on the numerical results for the experiments conducted. The results indicate that the six methods are sensitive to the initial points, which clearly affects the values of the three metrics considered in the experiments. All the six schemes are especially, sensitive to the initial points $x 2, x 4, x 6, x 7$ and $x 8$ as problems solved with them require more number of iterations, functions evaluations, mostly two or three digits, and processing time respectively. The eight initial points are particularly challenging for the MHZM1 and CGD schemes, where, for each of the initial points, the MHZM1 scheme fail to solve Problems 4.3, 4.4, 4.5 and 4.10, while the CGD scheme fail to solve Problems 4.1 and 4.2 for each of the starting points.

Furthermore, to conveniently access the impact of the choices of the HZ parameters on the new schemes, three figures are plotted by adopting the popular tool designed by Dolan and Moré [19], which can be expressed as

$$
\mathbf{p}_{w}(\varpi)=\frac{1}{|\mathcal{C}|}\left|\left\{s \in \mathcal{C}: \frac{t_{s, w}}{\min \left\{t_{s, w}: w \in W\right\}} \leq \varpi\right\}\right|,
$$

where $\mathcal{C}$ represent set of experiments conducted, $|\mathcal{C}|$ stands for number of the problems in the set of experiments $\mathcal{C}, W$ denotes number of schemes considered while for each $s \in \mathcal{C}$ and $w \in W, t_{s, w}$ represents either processing time in each iteration, iterations number or function values obtained. We begin with Figure 1, which highlights performances of each of the six schemes considered in the experiments with respect to number of iterations. The figure clearly shows that the NIHZPM and NEHZPM schemes outperform the MHZM1, MHZM2, EPGM and CGD schemes by solving more problems with much less number of iterations. In order to explain this, we employ the results from Tables 1 to 6 and the summary in Table 7. The summarized results indicate that the NIHZPM and NEHZPM methods alone, solved $10.42 \%$ and $22.57 \%$ of the problems with least number of iterations. However, simple computations from Tables 1 to 6 reveals that $37.11 \%$ and $36.54 \%$ of the problems were solved by the NIHZPM and NEHZPM methods and at least one of the other five methods with the least similar number of iterations. In each case, the two values sum up to $47.53 \%$ and $59.11 \%$, which are clearly captured by the right-hand side of the curves representing the NIHZPM and NEHZPM scheme on the vertical axis in Figure 1 respectively. The figure also shows that the NIHZPM and NEHZPM methods are more robust than the MHZM1, EPGM and CGD methods as the former's cumulative distribution function $\mathbf{p}(\varpi)$ got to 1 for minimal $\varpi$, while that of the latter stayed below 1 . On the other hand, the MHZM2 scheme competed well with the NIHZPM and NEHZPM schemes for $6.2 \leq \varpi \leq 8.2$. However, the NIHZPM and NEHZPM methods appear to be slightly more robust than the MHZM2 scheme. In a similar manner, the summary table and computations arising from results in Tables 1-6 involving problems, where ties are recorded by each method with at least one of the other five schemes, indicate that the MHZM1, MHZM2, EPGM and CGD schemes recorded 32.6\%, $27.43 \%, 17 \%$, and $18.84 \%$ as shown by their curves on the vertical axis in Figure 1 respectively. Next, we consider Figure 2, which displays performance profile of the six methods with regards to function evaluations. The summary table and computations from Tables 1 to 6 indicate that the NIHZPM, NEHZPM and MHZM2 methods are more superior to the other three methods with respect to function evaluations as the methods solved more problems with minimum function evaluations, which can be observed from the pattern displayed by their curves in Figure 2. As in the above case, Figure 2 indicate that both NIHZPM and NEHZPM methods are more robust, followed by the MHZM2 scheme, than the remaining three methods, as the former's cumulative distribution function $\mathbf{p}(\varpi)$ attain 1 for minimal $\varpi$, while the latter remained below 1 . Again, following similar approach, the summarized table and computations arising from results in Tables 1-6 involving problems, where ties are recorded by each method with at least one of the other five schemes, indicate that the MHZM1, EPGM and CGD schemes record $28.60 \%, 14.5 \%$, and $20 \%$ as exhibited by their curves on the vertical axis in Figure 2 respectively. It can be observed from the summary table that the EPGM method failed to solve any problem alone with the least function values. However, the scheme solved $14.5 \%$ of the problems with at least one of the 
five methods, which is clearly displayed by its curve in Figure 2. Lastly, Figure 3 shows that the NIHZPM and NEHZPM methods have an edge over the MHZM1, MHZM2, EPGM and CGD schemes, since the former solve more problems with less processing time than the latter. Careful inspection shows that the same values that are recorded in the summary table are displayed in Figure 3. In addition, Figure 3 shows that the NIHZPM, NEHZPM and MHZM2 are also more robust than the MHZM1, EPGM and CGD methods with respect to processing time.

Therefore, following the above discussion and analysis, it can be concluded that the NEHZPM and NIHZPM methods are better than the MHZM1, MHZM2, EPGM and CGD methods for solving system of monotone nonlinear equations of the reported collection.

\section{The Methods' APPlichtions IN COMPRESSED SENSING}

In this section, the NIHZPM and NEHZPM methods are applied to solve problems that often arise in compressed sensing; namely sparse signal reconstruction and blurred image restoration problems. Over the years, much effort have been made by researchers to obtain sparse solutions to under-determined or ill-conditioned linear systems of equations, which often arise in compressed sensing and other applications (see [20,22, 59, 80]). As a result, a number of iterative methods have been proposed to address the problem. The simplest approach involves minimizing a function with a quadratic $\ell_{2}$-norm error term and a sparseness including $\ell_{1}$-norm regularization term, which can be formulated as

$$
\min _{x} \frac{1}{2}\|H x-q\|_{2}^{2}+\varpi\|x\|_{1}
$$

where $x \in \mathbf{R}^{n}$ represents the signal to be reconstructed, $q \in \mathbf{R}^{l}$ denotes an observed data, $H \in \mathbf{R}^{l \times n}(l \ll n)$ is a linear operator, $\varpi$ is a regularization parameter, $\|x\|_{1}$ and $\|x\|_{2}$ are the $\ell_{1}$ and $\ell_{2}$ norms respectively. Clearly, (5.1) represents a convex unconstrained optimization problem, which is typically found in compressed sensing. So, an original signal, which is sparse or approximately sparse can be reconstructed exactly by solving (5.1).

A number of iterative methods are employed to solve (5.1) see [11,23,24,31], but the most popular are the gradient-based schemes, especially the gradient projection method for sparse reconstruction (GPSR) proposed by Figueiredo et al. [24]. Using this scheme, equation (5.1) is reformulated as a convex quadratic problem, where each vector $x \in \mathbf{R}^{n}$ is split in to two parts and presented as

$$
x=v-\nu, \quad v \geq 0, \quad \nu \geq 0, \quad v, \nu \in \mathbf{R}^{n} .
$$

with $v_{i}=\left(x_{i}\right)_{+}, \nu_{i}=\left(-x_{i}\right)_{+} \forall i=1,2, \ldots, n$ and $(.)_{+}=\max \{0, \cdot\}$. By the $\ell_{1}$-norm definition, $\|x\|_{1}=$ $E_{n}^{T} v+E_{n}^{T} \nu$, where $E_{n}=(1,1, \ldots, 1)^{T} \in \mathbf{R}^{n}$. Applying the above representation to (5.1), we obtain

$$
\min _{v, \nu} \frac{1}{2}\|H(v-\nu)-q\|_{2}^{2}+\varpi E_{n}^{T} v+\varpi E_{n}^{T} \nu,
$$

Also, according to Figueiredo et al. [24], the problem presented in (5.3) can be re-written as the following quadratic program problem

$$
\min _{s} \frac{1}{2} s^{T} B s+D^{T} B s, \quad s \geq 0
$$

where

$$
s=\left(\begin{array}{l}
v \\
\nu
\end{array}\right), \quad D=\varpi E_{2 n}+\left(\begin{array}{c}
-y \\
y
\end{array}\right), \quad y=H^{T} q, \quad B=\left(\begin{array}{cc}
H^{T} H & -H^{T} H \\
-H^{T} H & H^{T} H
\end{array}\right) .
$$

Since the matrix $B$ is positive semi-definite, it implies that (5.4) is a convex quadratic program problem. Quite recently, Xiao et al. [79] translated problem (5.4) into a linear variable inequality (LVI) problem, which they indicated is equivalent to a linear complementary problem, whose solution is $s$ if and only if it is a solution of the nonlinear equations given by

$$
g(s)=\min \{s, B s+D\}=0,
$$


Original $(n=4096$, number of nonzeros $=128)$
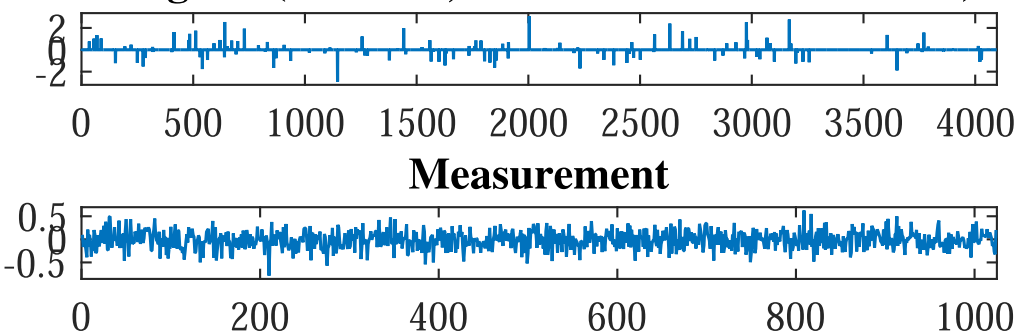

NEHZPM $($ MSE $=1.75 \mathrm{e}-05$, Iter $=165$, Time $=5.17 \mathrm{~s})$

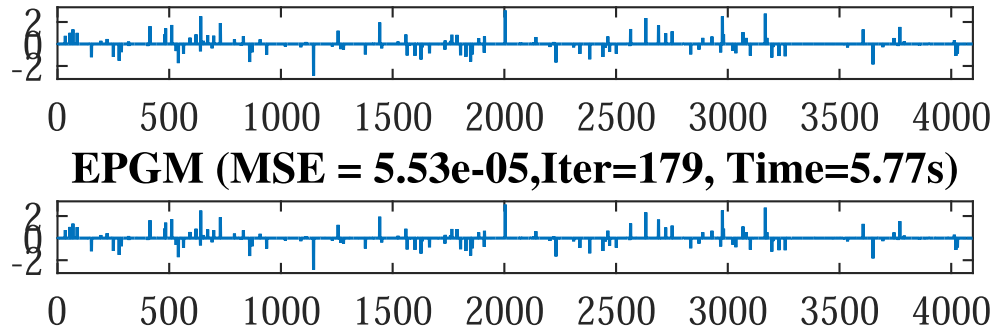

Figure 4. In descending order: Original signal, measurement, and recovery signals by NEHZPM and EPGM methods.

where $g$ represents a vector-valued mapping. Also, since $g$ is Lipschitz continuous and monotone (see $[53,79]$ ), it can be solved using the NIHZPM and NEHZPM schemes (Fig. 4).

\subsection{Sparse signal reconstruction with NEHZPM and EPGM methods}

In this subsection, some experiments are carried out to further demonstrate performance of the NEHZPM method. The focus is on a compressive sensing problem, where the target is reconstruction of a sparse signal with length $n$ from $l$ observations. The quality of restoration is measured by employing the mean square error (MSE) to the original signal $\hat{x}$, namely

$$
\mathrm{MSE}=\frac{1}{n}\left\|\hat{x}-x^{*}\right\|^{2},
$$

with $x^{*}$ representing the restored signal. In the experiment, we implemented the NEHZPM scheme with the parameters all set as used in the earlier numerical experiments. In addition, the size of the signal is set as $n=2^{12}$ and $l=2^{10}$, while the original signal contains randomly nonzero elements. Also, command $\operatorname{randn}(l, n)$ in Matlab generates the Gaussian matrix H. In this experiment, the measurement $q$ is disturbed by noise, i.e.,

$$
q=H \hat{x}+\omega,
$$

where $\omega$ denotes the Gaussian noise distributed as $N\left(0,10^{-4}\right)$. In order to test the performance of the NEHZPM scheme in signal restoration, it is compared with the EPGM method [39], which was recently applied to solve the same problem. The merit function is also given as $\psi(x)=\frac{1}{2}\|q-H x\|_{2}^{2}+\varpi\|x\|_{1}$.

As with the measure considered in [24], in this experiment the value of $\varpi$ is forced to decrease. Also, the iterative process for the experiment is started by the measurement signal i.e., $x_{0}=H^{T} q$, and terminates when the relative change between successive iterates fall below $10^{-5}$ i.e.,

$$
\frac{\left\|\psi_{k}-\psi_{k-1}\right\|}{\left\|\psi_{k-1}\right\|}<10^{-5},
$$

where $\psi_{k}$ denotes the function value at $x_{k}$. 
TABLE 8. Reported results of fifteen experiment with average for NEHZPM and EPGM methods on $\ell_{1}$-norm regularization problem.

\begin{tabular}{|c|c|c|c|c|c|c|c|c|}
\hline & \multicolumn{4}{|c|}{ NEHZPM } & \multicolumn{4}{|c|}{ EPGM } \\
\hline & ObjFun & Ptime & Inum & MSE & ObjFun & Ptime & Inum & MSE \\
\hline & $4.389 \times 10^{-1}$ & 5.17 & 165 & $1.751 \times 10^{-5}$ & $4.420 \times 10^{-1}$ & 5.77 & 179 & $5.532 \times 10^{-5}$ \\
\hline & $4.678 \times 10^{-1}$ & 4.30 & 140 & $1.899 \times 10^{-5}$ & $4.830 \times 10^{-1}$ & 5.23 & 162 & $9.049 \times 10^{-5}$ \\
\hline & $3.302 \times 10^{-1}$ & 4.92 & 159 & $1.407 \times 10^{-5}$ & $3.351 \times 10^{-1}$ & 5.73 & 176 & $5.370 \times 10^{-5}$ \\
\hline & $5.170 \times 10^{-1}$ & 4.86 & 159 & $2.220 \times 10^{-5}$ & $5.342 \times 10^{-1}$ & 5.59 & 173 & $1.142 \times 10^{-4}$ \\
\hline & $3.870 \times 10^{-1}$ & 4.75 & 155 & $1.547 \times 10^{-5}$ & $3.890 \times 10^{-1}$ & 5.83 & 175 & $4.180 \times 10^{-5}$ \\
\hline & $3.156 \times 10^{-1}$ & 5.23 & 161 & $1.232 \times 10^{-5}$ & $3.182 \times 10^{-1}$ & 5.86 & 179 & $4.103 \times 10^{-5}$ \\
\hline & $5.122 \times 10^{-1}$ & 4.73 & 150 & $2.331 \times 10^{-5}$ & $5.367 \times 10^{-1}$ & 5.42 & 164 & $1.672 \times 10^{-4}$ \\
\hline & $4.140 \times 10^{-1}$ & 5.50 & 173 & $1.925 \times 10^{-5}$ & $4.173 \times 10^{-1}$ & 6.11 & 192 & $6.206 \times 10^{-5}$ \\
\hline & $4.062 \times 10^{-1}$ & 5.20 & 161 & $1.780 \times 10^{-5}$ & $4.096 \times 10^{-1}$ & 6.00 & 178 & $5.912 \times 10^{-5}$ \\
\hline & $3.789 \times 10^{-1}$ & 5.03 & 161 & $1.516 \times 10^{-5}$ & $3.921 \times 10^{-1}$ & 5.17 & 166 & $9.400 \times 10^{-5}$ \\
\hline & $3.285 \times 10^{-1}$ & 5.22 & 171 & $1.273 \times 10^{-5}$ & $3.308 \times 10^{-1}$ & 6.39 & 208 & $4.025 \times 10^{-5}$ \\
\hline & $4.008 \times 10^{-1}$ & 5.03 & 166 & $1.816 \times 10^{-5}$ & $4.045 \times 10^{-1}$ & 5.58 & 183 & $6.289 \times 10^{-5}$ \\
\hline & $3.942 \times 10^{-1}$ & 5.14 & 164 & $1.853 \times 10^{-5}$ & $3.971 \times 10^{-1}$ & 5.97 & 191 & $5.689 \times 10^{-5}$ \\
\hline & $4.091 \times 10^{-1}$ & 4.75 & 161 & $1.582 \times 10^{-5}$ & $4.107 \times 10^{-1}$ & 5.50 & 178 & $4.087 \times 10^{-5}$ \\
\hline & $5.712 \times 10^{-1}$ & 5.00 & 155 & $2.692 \times 10^{-5}$ & $5.821 \times 10^{-1}$ & 5.47 & 172 & $1.113 \times 10^{-4}$ \\
\hline AVERAGE & $4.18 \times 10^{-1}$ & 4.988 & 160.06 & $1.78 \times 10^{-5}$ & $4.25 \times 10^{-1}$ & 5.708 & 178.40 & $7.693 \times 10^{-5}$ \\
\hline
\end{tabular}
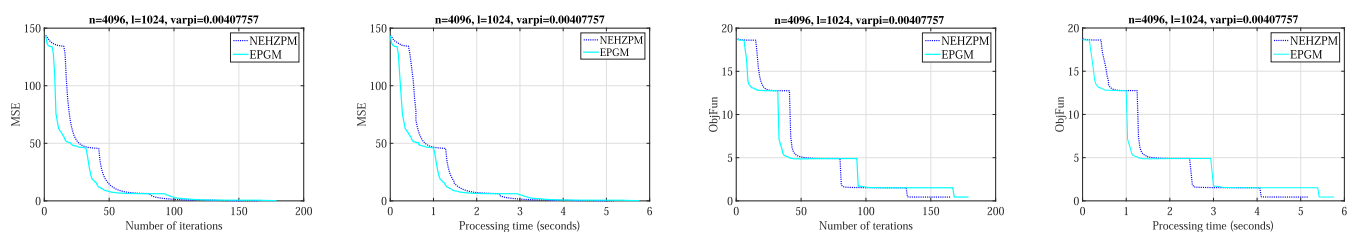

FiguRE 5. Comparison results of NEHZPM and EPGM methods. From the left, the horizontal axes of the first and third figures represent number of iterations while that of the second and fourth figures represent processing time in seconds. The vertical axes of the first and second figures represent the mean square error (MSE), while that of third and fourth figures represent the function values.

For the NEHZPM and EPGM schemes, we carried out fifteen experiments for different noise samples and report the results, which highlights the original sparse signal, the measurement as well as the reconstructed signal by each of the methods in Table 8. Also, four graphs are plotted to exhibit the convergence behavior of both methods through their mean square error (MSE) and function evaluations results, as well as number of iterations and processing time respectively. As can be observed from Figure 5, our proposed method exhibits much faster descent rates of MSE and function evaluations than the EPGM method. It can also be seen from the figures that the NEHZPM method requires less number of iterations and processing time in order to recover the original signal compared to that required by the EPGM method for the same process.

\subsection{Image restoration experiment with NIHZPM and CGD methods}

Here, some experiments are conducted with the NIHZPM and CGD methods to further highlight application and performance of the NIHZPM scheme. The experiment is on image restoration, which involves recovering or reconstructing an obscure or blurry image. Four different images are employed for the experiment, which includes, Einstein, Lena, Barbara and Cameraman. MATLAB R2014a is employed to generate all the codes 
TABLE 9. Performance results for NIHZPM and CGD methods based on objective function (ObjFun) value, mean square error (MSE), SNR and SSIM index.

\begin{tabular}{|c|c|c|c|c|c|c|c|c|}
\hline \multirow{2}{*}{$\begin{array}{l}\text { Image } \\
\text { size }\end{array}$} & \multicolumn{2}{|c|}{ ObjFun } & \multicolumn{2}{|c|}{ MSE } & \multicolumn{2}{|c|}{ SNR } & \multicolumn{2}{|c|}{ SSIM } \\
\hline & NIHZPM & CGD & NIHZPM & CGD & NIHZPM & CGD & NIHZPM & CGD \\
\hline $\begin{array}{l}\text { Einstein } \\
256 \times 256\end{array}$ & $4.132 \times 10^{6}$ & $4.049 \times 10^{6}$ & $7.9283 \times 10^{1}$ & $5.7765 \times 10^{1}$ & 20.94 & 22.32 & 0.78 & 0.82 \\
\hline $\begin{array}{l}\text { Lena } 256 \times \\
256\end{array}$ & $1.451 \times 10^{6}$ & $1.513 \times 10^{6}$ & $6.4591 \times 10^{1}$ & $9.0027 \times 10^{1}$ & 24.37 & 22.93 & 0.90 & 0.87 \\
\hline $\begin{array}{l}\text { Barbara } \\
256 \times 256\end{array}$ & $1.491 \times 10^{6}$ & $1.585 \times 10^{6}$ & $1.1545 \times 10^{2}$ & $2.0627 \times 10^{2}$ & 22.07 & 19.55 & 0.84 & 0.75 \\
\hline $\begin{array}{l}\text { Cameraman } \\
256 \times 256\end{array}$ & $1.394 \times 10^{6}$ & $1.473 \times 10^{6}$ & $1.1335 \times 10^{2}$ & $1.7757 \times 10^{2}$ & 22.00 & 20.05 & 0.88 & 0.83 \\
\hline
\end{tabular}

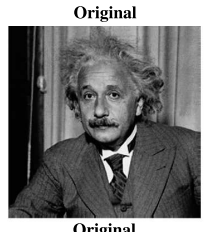

Original

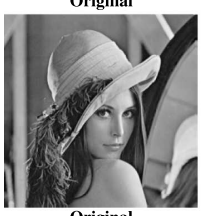

Original

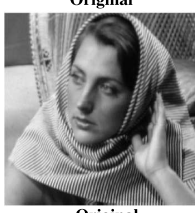

Original

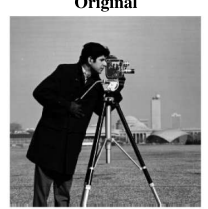

Blurred

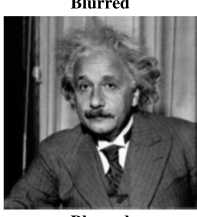

Blurred

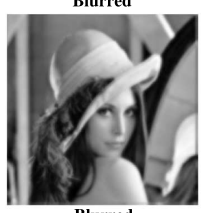

Blurred

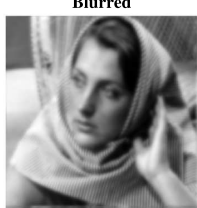

Blurred

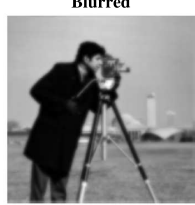

NIHZPM

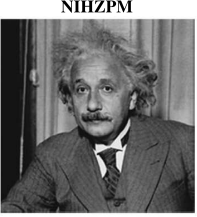

NIHZPM

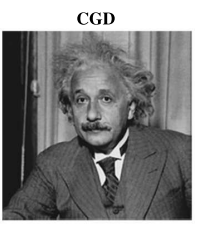

CGD

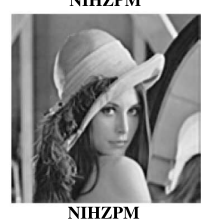

NIHZPM
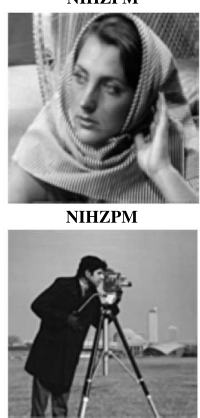

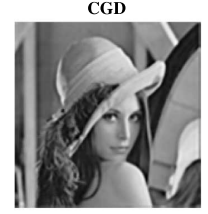

CGD

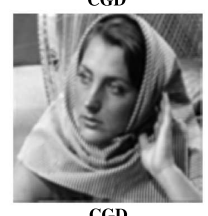

CGD

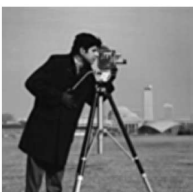

Figure 6. Original and blurred images (First two columns from the left). Restored images by the two methods (Third and Fourth columns).

with the same configuration and parameter values as used in the last experiment with $\gamma=0.6$. Also, to obtain an insight into the performance of the NIHZPM method, it is compared with CGD [78] solver, which is used in image restoration problem. The parameters for this method are the same as used by the authors. The performance of both schemes in terms of number of iterations (Niter), processing time (PT(s)), mean square error (MSE), signal to noise ratio (SNR), which is given by

$$
\mathrm{SNR}=20 \times \log _{10}\left(\frac{\|\hat{x}\|}{\|x-\hat{x}\|}\right),
$$

where $\hat{x}$ represents the recovered image, $x$ denotes the original image, and the structural similarity index (SSIM), which computes the similarity between original image and the restored one in each of the experiments 
conducted. In the experiment, $H$ represents a partial Discrete Wavelet Transform (DWT) matrix, for which the $m$ rows are selected randomly from the $n \times n$ DWT matrix. The encoding matrix $H$ is able to be tested on large images without storing any matrix, since it doesn't require storage and also enables fast matrix-vector multiplications involving $H$ and $H^{T}$. Results of the experiments conducted are presented in Table 9, while Figure 6 displays the original, blurred, and reconstructed images obtained by the NIHZPM and CGD schemes. It can be observed from Table 9 that the NIHZPM scheme has an edge over the CGD scheme in all the four metrics considered, namely objective function (ObjFun), mean square error (MSE), signal to noise ratio (SNR) and structural similarity index (SSIM). Figure 6 also show that, for the exception of Einstein, images restored from the blurred images by NIHZPM scheme appears slightly closer to the original one than the restored images by CGD method. Hence, based on this analysis, it can be concluded that the NIHZPM method is suitable for reconstruction of the images considered. The MATLAB implementation of the SSIM index can be obtained at http://www.cns.nyu.edu/lcv/ssim/.

\section{Conclusion}

Two effective algorithms for solving systems of nonlinear monotone equations with convex constraint are presented in this article. The schemes are based on the one-parameter Hager-Zhang method for unconstrained optimization. By carrying out eigenvalue study of a modified Hager-Zhang search direction matrix, and singular value analysis of its revised symmetric version, two new Hager-Zhang search directions are derived, which are combined with the projection technique. An attractive feature of the proposed methods is that they require low memory to implement, which is an attribute that qualifies them to solve large scaled problems. The schemes also solve nonsmooth nonlinear equations, since they are derivative-free. By applying basic conditions, we proved global convergence of the methods proposed and numerical results of experiments carried out show that both methods perform much better than the two recently proposed Hager-Zhang schemes and two other methods in the literature. To further demonstrate the effectiveness of the new methods, they are applied to solve signal and image reconstruction problems in compressed sensing. Finally, as a future research, we anticipate extending modified versions of the methods proposed to solve motion control problems in engineering and other real life applications.

Acknowledgements. The authors would like to express their immense gratitude to the anonymous reviewers for their comments as well as suggestions that have really helped in improving quality of the work. We would also like to thank the entire members of the numerical optimization research group, Bayero university, Kano for their encouragement in the course of this work.

Conflict of interest. The authors declare that they have no conflict of interest.

Data availability statement. The authors confirm that, apart from the MATLAB implementation of the SSIM index, which can be obtained at http://www.cns.nyu.edu/lcv/ssim/, all data generated or analysed during this study are included in this article.

\section{REFERENCES}

[1] H. Abdullahi, A.S. Halilu and M.Y. Waziri, A modified conjugate gradient method via a double direction approach for solving large-scale symmetric nonlinear equations. J. Numer. Math. Stoch. 10 (2018) 32-44.

[2] A.B. Abubakar, P. Kumam, H. Mohammad, A.M. Awwal and K. Sitthithakerngkiet, A modified Fletcher-Reeves conjugate gradient method for monotone nonlinear equations with some applications. Mathematics 7 (2019) 745.

[3] N. Andrei, Open problems in conjugate gradient algorithms for unconstrained optimization. Bull. Malays. Math. Sci. Soc. 34 (2011) 319-330.

[4] N. Andrei, Accelerated adaptive Perry conjugate gradient algorithms based on the self-scaling BFGS update. J. Comput. Appl. Math. 325 (2017) 149-164.

[5] N. Andrei, A note on memory-less SR1 and memory-less BFGS methods for large-scale unconstrained optimization. Numer. Algor. (2021). DOI: 10.1007/s11075-021-01185-8.

[6] M.R. Arazm, S. Babaie-Kafaki and R. Ghanbari, An extended Dai-Liao conjugate gradient method with global convergence for nonconvex functions. Glasnik Matematic 52 (2017) 361-375. 
[7] S. Babaie-Kafaki and R. Ghanbari, A descent family of Dai-Liao conjugate gradient methods. Optim. Methods Softw. 29 (2013) 583-591.

[8] S. Babaie-Kafaki and R. Ghanbari, The Dai-Liao nonlinear conjugate gradient method with optimal parameter choices. Eur. J. Oper. Res. 234 (2014) 625-630.

[9] S. Babaie-Kafaki and R. Ghanbari, Two optimal Dai-Liao conjugate gradient methods. Optimization 64 (2015) $2277-2287$.

[10] S. Babaie-Kafaki, R. Ghanbari and N. Mahdavi-Amiri, Two new conjugate gradient methods based on modified secant equations. J. Comput. Appl. Math. 234 (2010) 1374-1386.

[11] A. Beck and M. Teboulle, A fast iterative shrinkage-thresholding algorithm for linear inverse problems. SIAM J. Imag. Sci. 2 (2009) 183-202.

[12] C.G. Broyden, A class of methods for solving nonlinear simultaneous equations. Math. Comput. 19 (1965) $577-593$.

[13] W. Cheng, A PRP type method for systems of monotone equations. Math. Comput Modelling 50 (2009) 15-20.

[14] Y.H. Dai and L.Z. Liao, New conjugacy conditions and related nonlinear conjugate gradient methods. Appl. Math. Optim. 43 (2001) $87-101$.

[15] Y.H. Dai and Y.X. Yuan, Nonlinear Conjugate Gradient Methods. Shanghai Scientific and Technical Publishers, Shanghai (2000).

[16] Z. Dai, X. Chen and F. Wen, A modified Perry's conjugate gradient method-based derivative-free method for solving large-scale nonlinear monotone equation. Appl. Math. Comput. 270 (2015) 378-386.

[17] M.K. Dauda, M. Mamat, M.A. Mohamed and M.Y. Waziri, Improved quasi-Newton method via SR1 update for solving symmetric systems of nonlinear equations. Malay. J. Fund. Appl. Sci. 15 (2019) 117-120.

[18] Y. Ding, Y. Xiao and J. Li, A class of conjugate gradient methods for convex constrained monotone equations, Optimization 66 (2017) 2309-2328.

[19] E.D. Dolan and J.J. Moré, Benchmarking optimization software with performance profiles. Math. Program. 91 (2002) $201-213$.

[20] M. Elad, Sparse and Redundant Representations: From Theory to Applications in Signal and Image Processing. Springer Science and Business Media, LCC, Berlin (2010).

[21] M. Fatemi, An optimal parameter for Dai-Liao family of conjugate gradient methods. J. Optim. Theory Appl. 169 (2016) $587-605$.

[22] J.A. Fessler, Model-based image reconstruction for MRI. IEEE Signal Process. Mag. 27 (2010) 81-89.

[23] M.A.T. Figueiredo and R. Nowak, An EM algorithm for wavelet-based image restoration. IEEE Trans. Image Process. 12 (2003) 906-916.

[24] M. Figueiredo, R. Nowak and S.J. Wright, Gradient projection for sparse reconstruction, application to compressed sensing and other inverse problems. In: IEEE J-STSP. IEEE Press, Piscataway, NJ (2007) 586-597.

[25] J.A. Ford and I.A. Moghrabi, Multi-step quasi-Newton methods for optimization. J. Comput. Appl. Math. 50 (1994) $305-323$.

[26] J.A. Ford, Y. Narushima and H. Yabe, Multi-step nonlinear conjugate gradient methods for unconstrained minimization. Comput. Optim. Appl. 40 (2008) 191-216.

[27] L. Grippo, F. Lampariello and S. Lucidi, A nonmonotone line search technique for Newton's method. SIAM J. Numer. Anal. 23 (1986) $707-716$.

[28] W.W. Hager and H. Zhang, A new conjugate gradient method with guaranteed descent and an efficient line search. SIAM J. Optim. 16 (2005) 170-192.

[29] W.W. Hager and H. Zhang, A survey of nonlinear conjugate gradient methods. Pac. J. Optim. 2 (2006) 35-58.

[30] W.W. Hager and H. Zhang, Algorithm 851: CG_Descent, a conjugate gradient method with guaranteed descent. ACM Trans. Math. Softw. 32 (2006) 113-137.

[31] E.T. Hale, W. Yin and Y. Zhang, A fixed-point continuation method for $\ell_{1}$ regularized minimization with applications to compressed sensing. SIAM J. Optim. 19 (2008) 1107-1130.

[32] A.S. Halilu and M.Y. Waziri, An improved derivative-free method via double direction approach for solving systems of nonlinear equations. J. Ram. Mathl. Soc. 33 (2018) 75-89.

[33] A.S. Halilu, A. Majumder, M.Y. Waziri and H. Abdullahi, Double direction and step length method for solving system of nonlinear equations. Eur. J. Mol. Clinl. Med. 7 (2020) 3899-3913.

[34] A.S. Halilu, A. Majumder, M.Y. Waziri, A.M. Awwal and K. Ahmed, On solving double direction methods for convex constrained monotone nonlinear equations with image restoration. Comput. Appl. Math. 40 (2021) 1-27.

[35] A.S. Halilu, A. Majumder, M.Y. Waziri, K. Ahmed and A.M. Awwal, Motion control of the two joint planar robotic manipulators through accelerated Dai-Liao method for solving system of nonlinear equatiions. Eng. Comput. (2021). DOI: 10.1108/EC-06-2021-0317.

[36] A.S. Halilu, A. Majumder, M.Y. Waziri and K. Ahmed, Signal recovery with convex constrained nonlinear monotone equations through conjugate gradient hybrid approach. Math. Comp. Simul. 187 (2021) 520-539.

[37] M.R. Hestenes and E.L. Stiefel, Methods of conjugate gradients for solving linear systems. J. Res. Nat. Bur. Stand. 49 (1952) 409-436.

[38] G.A. Hively, On a class of nonlinear integral equations arising in transport theory. SIAM J. Math. Anal. 9 (1978) $787-792$.

[39] Y. Hu and Y. Wang, An efficient projected gradient method for convex constrained monotone equations with applications in compressive sensing. J. Appl. Math. Phys. 8 (2020) 983-998.

[40] Z. Khoshgam and A. Ashrafi, A new modidified scaled conjugate gradient method for large-scale unconstrained optimization with non-convex objective function. Optim. Methods Softw. 34 (2019) 783-796. 
[41] M.S. Koorapetse and P. Kaelo, Globally convergent three-term conjugate gradient projection methods for solving nonlinear monotone equations. Arab. J. Math. 7 (2018) 289-301.

[42] W. La Cruz, A spectral algorithm for large-scale systems of nonlinear monotone equations. Numer. Algor. 76 (2017) 1109-1130.

[43] W. La Cruz, J.M. Martinez and M. Raydan, Spectral residual method without gradient information for solving large-scale nonlinear systems of equations: theory and experiments. Technical Report RT-04-08 (2004).

[44] D.H. Li and M. Fukushima, A derivative-free line search and global convergence of Broyden-like method for nonlinear equations. Optim. Methods Softw. 13 (2000) 583-599.

[45] D.H. Li and M. Fukushima, A globally and superlinearly convergent Gauss-Newton-based BFGS method for symmetric nonlinear equations. SIAM J. Numer. Anal. 37 (2000) 152-172.

[46] J.K. Liu and S.J. Li, A projection method for convex constrained monotone nonlinear equations with applications. Comput. Math. Appl. 70 (2015) 2442-2453.

[47] D.Y. Liu and Y.F. Shang, A new Perry conjugate gradient method with the generalized conjugacy condition. In: Comput. Intel. Softw. Eng. (CiSE). 2010 International Conference on Issue (2010).

[48] D.Y. Liu and G.Q. Xu, A Perry descent conjugate gradient method with restricted spectrum. Optimization Online, Nonlinear Optimization (unconstrained optimization) (2011) 1-19.

[49] I.E. Livieris and P. Pintelas, Globally convergent modified Perrys conjugate gradient method. Appl. Math. Comput. 218 (2012) 9197-9207.

[50] Y.B. Musa, M.Y. Waziri and A.S. Halilu, On computing the regularization parameter for the Levenberg-Marquardt method via the spectral radius approach to solving systems of nonlinear equations. J. Numer. Math. Stoch. 9 (2017) 80-94.

[51] J. Nocedal and S.J. Wright, Numerical Optimization. Springer, New York (1999).

[52] J.M. Ortega and W.C. Rheinboldt, Iterative Solution of Nonlinear Equations in Several Variables. Academic Press, New York (1970).

[53] J.S. Pang, Inexact Newton methods for the nonlinear complementarity problem. Math. Program. 36 (1986) $54-71$.

[54] G. Peiting and H. Chuanjiang, A derivative-free three-term projection algorithm involving spectral quotient for solving nonlinear monotone equations. Optimization 67 (2018) 1631-1648.

[55] J.M. Perry, A class of conjugate gradient algorithms with a two step variable metric memory. Discussion paper 269, Center for Mathematical Studies in Economics and Management Science, Northwestern University, Chicago (1977).

[56] A. Perry, A modified conjugate gradient algorithm. Oper. Res. Tech. Notes 26 (1978) 1073-1078.

[57] B.T. Polak, The conjugate gradient method in extreme problems. USSR Comput. Math. Math. Phys. 4 (1969) $94-112$.

[58] E. Polak and G. Ribičre, Note sur la convergence de méthodes de directions conjuguées. Rev. Fr. Inf. Rech. Oper. 16 (1969) $35-43$.

[59] J.K. Romberg, Imaging via compressive sampling. IEEE Signal Process. Mag. 25 (2008) 14-20.

[60] J. Sabi'u, A. Shah and M.Y. Waziri, Two optimal Hager-Zhang conjugate gradient methods for solving monotone nonlinear equations. Appl. Numer. Math. 153 (2020) 217-233.

[61] J. Sabi'u, A. Shah, M.Y. Waziri and K. Ahmed, Modified Hager-Zhang conjugate gradient methods via singular value analysis for solving monotone nonlinear equations with convex constraint. Int. J. Comput. Meth. 18 (2021) 2050043.

[62] D.F. Shanno, On the convergence of a new conjugate gradient algorithm. SIAM J. Numer. Anal. 15 (1978) $1247-1257$.

[63] M.V. Solodov and A.N. Iusem, Newton-type methods with generalized distances for constrained optimization. Optimization 41 (1997) 257-278.

[64] M.V. Solodov and B.F. Svaiter, A globally convergent inexact Newton method for systems of monotone equations. In: Reformulation: Nonsmooth, Piecewise Smooth, Semismooth and Smoothing Methods. Springer (1998) 355-369.

[65] W. Sun and Y.X. Yuan, Optimization Theory and Methods: Nonlinear Programming. Springer, New York (2006).

[66] S. Wang and H. Guan, A scaled conjugate gradient method for solving monotone nonlinear equations with convex constraints. J. Appl. Math. (2013). DOI: 10.1155/2013/286486.

[67] D.S. Watkins, Fundamentals of Matrix Computations, 2nd edition. John Wiley and Sons, New York (2004).

[68] M.Y. Waziri and K. Ahmed, Two descent Dai-Yuan conjugate gradient methods for systems of monotone nonlinear equations. J. Sci. Comput. 90 (2022) 1-53.

[69] M.Y. Waziri, W.J. Leong and M.A. Hassan, Jacobian free-diagonal Newton's method for nonlinear systems with singular Jacobian. Malay. J. Math. Sci. 5 (2011) 241-255.

[70] M.Y. Waziri, K. Ahmed and J. Sabi'u, A family of Hager-Zhang conjugate gradient methods for system of monotone nonlinear equations. Appl. Math. Comput. 361 (2019) 645-660.

[71] M.Y. Waziri, K. Ahmed and J. Sabi'u, Descent Perry conjugate gradient methods for systems of monotone nonlinear equations. Numer. Algor. 85 (2020) 763-785.

[72] M.Y. Waziri, K. Ahmed and J. Sabi'u, A Dai-Liao conjugate gradient method via modified secant equation for system of nonlinear equations. Arab. J. Math. 9 (2020) 443-457.

[73] M.Y. Waziri, K. Ahmed, J. Sabi'u and A.S. Halilu, Enhanced Dai-Liao conjugate gradient methods for systems of monotone nonlinear equations. SeMA J. 78 (2020) 15-51.

[74] M.Y. Waziri, Y.M. Kufena and A.S. Halilu, Derivative-free three-term spectral conjugate gradient method for symmetric nonlinear equations. Thai J. Math. 18 (2020) 1417-1431.

[75] M.Y. Waziri, K. Ahmed, A.S. Halilu and A.M. Awwal, Modified Dai-Yuan iterative scheme for nonlinear systems and its application. Numer. Alg. Control Optim. (2021). DOI: 10.3934/naco.2021044. 
[76] M.Y. Waziri, H. Usman, A.S. Halilu and K. Ahmed, Modified matrix-free methods for solving systems of nonlinear equations. Optimization 70 (2021) 2321-2340.

[77] M.Y. Waziri, Y.M. Kufena and A.S. Halilu, Double direction three-term spectral conjugate gradient method for solving symmetric nonlinear equations. Res. Control Opt. 6 (2022) 1-11.

[78] Y.H. Xiao and H. Zhu, A conjugate gradient method to solve convex constrained monotone equations with applications in compressive sensing. J. Math. Anal. Appl. 405 (2013) 310-319.

[79] Y.H. Xiao, Q.Y. Wang and Q.J. Hu, Non-smooth equations based method for $\ell_{1}$-norm problems with applications to compressed sensing. Nonlinear Anal. TMA 74 (2011) 3570-3577.

[80] J.C. Yang, J. Wright, T.S. Huang and Y. Ma, Image super-resolution via sparse representation. IEEE Trans. Image Process. 19 (2010) 2861-2873.

[81] G. Yu, A derivative-free method for solving large-scale nonlinear systems of equations. J. Ind. Manag. Optim. 6 (2010) 149-160.

[82] Z. Yu, J. Lina, J. Sun, Y. Xiao, L. Liu and Z. Li, Spectral gradient projection method for monotone nonlinear equations with convex constraints. Appl. Numer. Math. 59 (2009) 2416-2424.

[83] N. Yuan, A derivative-free projection method for solving convex constrained monotone equations. ScienceAsia 43 (2017) 195-200.

[84] G. Yuan, B. Wang and Z. Sheng, The Hager-Zhang conjugate gradient algorithm for large-scale nonlinear equations, Int. J. Comput. Math. 96 (2019) 1533-1547.

[85] L. Zhang and W. Zhou, Spectral gradient projection method for solving nonlinear monotone equations. J. Comput. Appl. Math. 196 (2006) 478-484.

[86] Y.B. Zhao and D. Li, Monotonicity of fixed point and normal mappings associated with variational inequality and its application. SIAM J. Optim. 11 (2001) 962-973.

[87] W. Zhou and D. Li, Limited memory BFGS method for nonlinear monotone equations. J. Comput. Math. 25 (2007) 89-96.

\section{Subscribe to Open (S20) A fair and sustainable open access model}

This journal is currently published in open access under a Subscribe-to-Open model (S2O). S2O is a transformative model that aims to move subscription journals to open access. Open access is the free, immediate, online availability of research articles combined with the rights to use these articles fully in the digital environment. We are thankful to our subscribers and sponsors for making it possible to publish this journal in open access, free of charge for authors.

\section{Please help to maintain this journal in open access!}

Check that your library subscribes to the journal, or make a personal donation to the $\mathrm{S} 2 \mathrm{O}$ programme, by contacting subscribers@edpsciences.org

More information, including a list of sponsors and a financial transparency report, available at: https://www. edpsciences.org/en/maths-s2o-programme 